\title{
Modelling of the ultraviolet and visual SED variability in the hot magnetic Ap star CU Virginis
}

\author{
J. Krtička ${ }^{1}$, Z. Mikulášek ${ }^{1,2}$, T. Lüftinger ${ }^{3}$, D. Shulyak ${ }^{4}$, J. Zverko ${ }^{5}$, J. Žižňovský ${ }^{6}$, and N. A. Sokolov ${ }^{7}$ \\ ${ }^{1}$ Department of Theoretical Physics and Astrophysics, Masaryk University, Kotlářská 2, 61137 Brno, Czech Republic \\ e-mail: krticka@physics.muni.cz \\ 2 Observatory and Planetarium of J. Palisa, VŠB - Technical University, Ostrava, Czech Republic \\ 3 Institut für Astronomie, Universität Wien, Türkenschanzstraße 17, 1180 Wien, Austria \\ ${ }^{4}$ Institute of Astrophysics, Georg-August-University, Friedrich-Hund-Platz 1, 37077 Göttingen, Germany \\ 5 Tatranská Lomnica 133, 05960, Slovak Republic \\ 6 Astronomical Institute, Slovak Academy of Sciences, Tatranská Lomnica, 05960, Slovak Republic \\ ${ }^{7}$ Central Astronomical Observatory at Pulkovo, St. Petersburg 196140, Russia
}

Received 16 June 2011 / Accepted 20 October 2011

\section{ABSTRACT}

\begin{abstract}
Context. The spectral energy distribution (SED) in chemically peculiar stars may be significantly affected by their abundance anomalies. The observed SED variations are usually assumed to be a result of inhomogeneous surface distribution of chemical elements, flux redistribution and stellar rotation. However, the direct evidence for this is still only scarce.

Aims. We aim to identify the processes that determine the SED and its variability in the UV and visual spectral domains of the heliumweak star CU Vir.

Methods. We used the TLUSTY model atmospheres calculated for the appropriate surface chemical composition to obtain the emergent flux and predict the rotationally modulated flux variability of the star.

Results. We show that most of the light variations in the $v b y$ filters of the Strömgren photometric system are a result of the uneven surface distribution of silicon, chromium, and iron. Our models are only able to explain a part of the variability in the $u$ filter, however. The observed UV flux distribution is very well reproduced, and the models are able to explain most of the observed features in the UV light curve, except for the region 2000-2500 ̊, where the amplitude of the observed light variations is higher than predicted. The variability observed in the visible is merely a faint gleam of that in the UV. While the amplitude of the light curves reaches only several hundredths of magnitude in the visual domain, it reaches about 1 mag in the UV.

Conclusions. The visual and UV light variability of CU Vir is caused by the flux redistribution from the far UV to near UV and visible regions, inhomogeneous distribution of the elements and stellar rotation. Bound-free transitions of silicon and bound-bound transitions of iron and chromium contribute the most to the flux redistribution. This mechanism can explain most of the rotationally modulated light variations in the filters centred on the Paschen continuum and on the UV continuum of the star CU Vir. However, another mechanism(s) has to be invoked to fully explain the observed light variations in the $u$ filter and in the region 2000-2500 Å.
\end{abstract}

Key words. stars: chemically peculiar - stars: early type - stars: atmospheres - stars: variables: general - stars: individual CU Virginis

\section{Introduction}

Chemically peculiar (CP) stars are among the most enigmatic objects of the upper part of the main-sequence. The processes of radiative diffusion and gravitational settling in the atmospheres of these stars cause pronounced deviations from the solar value in the chemical composition (Vauclair 2003, Michaud 2004). Apart from the chemical peculiarity, many of CP stars show variations in the magnetic field as well as in their spectra and light. These variations are usually strictly periodical and modulated by the rotation of the star. The uneven distribution of the surface magnetic field (with dipole component dominating in most cases) is one of the factors that cause the uneven distribution of chemical elements and, consequently, also the periodic spectrum variability. The uneven distribution of chemical elements is suspected to be the origin of the light variability, but this connection is still not very well understood.

The line blanketing caused by numerous lines of overabundant elements (mainly the iron-peak ones) and the flux redistribution induced by these lines has been suspected to significantly affect the spectral energy distribution (SED) and to be the prime source of the light variability (e.g., Molnar 1973; Kodaira 1973). Bound-free transitions (Peterson 1970; Lanz et al. 1996) were also expected to play some role. Surface temperature differences or variable temperature gradients (Weiss et al. 1976; Stępień 1978) were on the list of possible causes of the SED variability as well. The presence of a magnetic field may affect the SED and its variability, provided the field is sufficiently strong (Kochukhov et al. 2005). Finally, hot CP stars may have circumstellar shells fed by the stellar wind, causing variability through the light absorption (Landstreet \& Borra 1978; Nakajima 1985; Smith \& Groote 2001; Townsend et al. 2005).

Realistic SED simulations of CP stars were not possible until the techniques of Doppler mapping and model atmosphere calculations were considerably developed. The Doppler mapping enables one to precisely map the distribution of individual elements on the surface of rotating stars (e.g., Rice et al. 1989; Khokhlova et al. 2000; Piskunov \& Kochukhov 2002; Lüftinger et al. 2010; Bohlender et al. 2010). Detailed model atmospheres (e.g., Lanz \& Hubeny 2007) enable one to precisely predict the 
fluxes from surface elements with a peculiar chemical composition, taking into account realistic bound-free (Seaton et al. 1992) and bound-bound (Kurucz 1994) transitions.

Based on precise model atmospheres and detailed surface maps it was possible to follow earlier attempts of light curve modelling (Krivosheina et al. 1980; Ryabchikova 1990) and to successfully simulate the light curves of several CP stars. Krtička et al. (2007) showed that the light variations of $\operatorname{HD} 37776\left(T_{\mathrm{eff}}=\right.$ $22000 \mathrm{~K})$ can be explained to be a result of inhomogeneous surface distribution of helium and silicon. Krtička et al. (2009) showed that most of the observed light variations in HR 7224 $\left(T_{\text {eff }}=14500 \mathrm{~K}\right)$ are caused by inhomogeneous surface distribution of silicon and iron. For the cooler star $\varepsilon \mathrm{UMa}\left(T_{\mathrm{eff}}=\right.$ $9000 \mathrm{~K})$ Shulyak et al. (2010a) showed that chromium can also contribute significantly to the light variability. Moreover, modern atmosphere models are able to explain the observed SED in detail (e.g., Shulyak et al. 2010b), pointing to the importance of rare-earth elements in cooler CP stars.

For our present study we selected one of the most enigmatic CP stars, CU Vir (HR 5313, HD 124224). The light variability of CU Vir has been known for more than half a century (Hardie 1956). CU Vir belongs to a rare group of CP stars that show period changes (Pyper et al. 1998; Pyper \& Adelman 2004; Trigilio et al. 2008, 2011; Mikulášek et al. 2011), theoretically studied by Stępień (1998). Moreover, CU Vir is a source of variable radio emission, resembling a radio lighthouse of pulsars (Trigilio et al. 2000; Kellett et al. 2007).

All these observations make CU Vir one of the most appealing targets for theoretical studies. We studied the nature of the SED variations of this star using Doppler abundance maps of Kuschnig et al. (1999).

\section{Simulation of the SED variability}

\subsection{Stellar parameters}

The stellar parameters of CU Vir and abundance maps of helium, silicon, chromium, and iron adopted from Kuschnig et al. (1999) are given in Table 1 . Note that there is also a magnesium abundance map available in Kuschnig et al. (1999), but because of the low maximum magnesium abundance derived we did not include its inhomogeneous surface distribution. The abundances in the maps are expressed as $\log \left(N_{\mathrm{el}} / N_{\text {tot }}\right)$, but we used abundances relative to hydrogen, i.e., $\varepsilon_{\mathrm{el}}=\log \left(N_{\mathrm{el}} / N_{\mathrm{H}}\right)$.

The calculation of the rotational phases for individual observations is not a straightforward task, because the instant rotational period $P(t)$ of the surface layers of CU Vir is changing. Accordingly, we applied the new ephemeris of Mikulášek et al. (2011), where the phase function $\vartheta(t)$ is approximated by the fourth-order polynomial of time:

$$
\begin{aligned}
& \vartheta(t) \cong \vartheta_{0}-\frac{A}{P_{0}}\left(\frac{3}{2} \Theta^{2}-\Theta^{4}\right) ; \vartheta_{0}=\frac{t-M_{0}}{P_{0}}, \Theta=\frac{t-T_{0}}{\Pi}, \\
& P(t)=1 / \dot{\vartheta} \doteq P_{0}\left[1+A / \Pi\left(3 \Theta-4 \Theta^{3}\right)\right]
\end{aligned}
$$

where $P(t)$ is the instant period at the time $t, \vartheta_{0}$ is the phase function for a linear ephemeris with the origin at $M_{0} \equiv$ 2446730.4447 and the basic period $P_{0}$. Mikulášek et al. (2011) found that $P_{0}=0.52069415(8), A=0.5643(29), \Pi=$ $13260(70) \mathrm{d}$, and $T_{0}=2446636(24)$. The formula accounts for the period variability observed in CU Vir and enables us to determine the rotational phase with an accuracy better than $0.002 P$.

The phase shift between this ephemeris $\phi=\operatorname{frac}(\vartheta(t))$ (fractional part of $\vartheta(t))$ and that used by Kuschnig et al. (1999) $\phi_{\text {Kus }}$
Table 1. CU Vir parameters from spectroscopy (Kuschnig et al. 1999).

\begin{tabular}{lc}
\hline \hline Effective temperature $T_{\text {eff }}$ & $13000 \mathrm{~K}$ \\
Surface gravity $\log g(\mathrm{cgs})$ & 4.0 \\
Inclination $i$ & $30^{\circ}$ \\
Rotational velocity projection $v_{\mathrm{rot}} \sin i$ & $160 \mathrm{~km} \mathrm{~s}^{-1}$ \\
Helium abundance & $-3.2<\varepsilon_{\mathrm{He}}<-1$ \\
Silicon abundance & $-4.6<\varepsilon_{\mathrm{Si}}<-2.3$ \\
Chromium abundance & $-6.6<\varepsilon_{\mathrm{Cr}}<-4.4$ \\
Iron abundance & $-5.5<\varepsilon_{\mathrm{Fe}}<-3.5$ \\
\hline
\end{tabular}

in the time of their spectral observations was $\Delta \phi=\phi-\phi_{\text {Kus }}=$ 0.52045 , and the origin of the phase function was determined to be at HJD 2446730.4447.

\subsection{Model atmospheres and synthetic spectra}

We used the code TLUSTY for the model atmosphere calculations (Hubeny 1988; Hubeny \& Lanz 1992, 1995; Lanz \& Hubeny 2003). Although the code enables us to calculate NLTE models, we confined ourselves to the LTE plane-parallel models, because we expected the NLTE effects to be marginal for the light variability. The atomic data (taken from Lanz \& Hubeny 2007) were selected to be appropriate for B type stars, the atomic data for silicon in particular are based on Mendoza et al. (1995), Butler et al. (1993), and Taylor (2011), in preparation ${ }^{1}$; for iron on Kurucz (1994), Nahar (1996), Nahar (1997), Bautista \& Pradhan (1997), and Bautista (1996), and for other elements on Luo \& Pradhan (1989), Fernley et al. (1999), Tully et al. (1990), Peach et al. (1988), Hibbert \& Scott (1994), and Nahar \& Pradhan (1993). We prepared our own ionic models for chromium (Cr II-Cr vI) using data taken from Kurucz (2009) ${ }^{2}$.

We assumed fixed values of the effective temperature and surface gravity (according to Table 1) and adopted a generic value of the microturbulent velocity $v_{\text {turb }}=2 \mathrm{~km} \mathrm{~s}^{-1}$. The abundance of helium, silicon, chromium, and iron differed in individual models as explained below. We used the solar abundance of other elements (Asplund et al. 2005).

For the calculation of synthetic spectra we used the SYNSPEC code. The synthetic spectra were calculated for the same parameters (effective temperature, surface gravity, and chemical composition) as the model atmospheres. We also took into account the same transitions as for the model atmosphere calculations. To this end we included the same chromium and iron lines as we used for the model atmosphere calculation in our SYNSPEC line list. This is not particularly important in the visible, but in the ultraviolet (UV) numerous lines for which only the theoretical data are available significantly influence the spectral energy distribution. Additionally, we included the lines of all elements with the atomic number $Z \leq 30$, that were not accounted fot the model atmosphere calculation. We computed angle-dependent intensities for 20 equidistantly spaced values of $\mu=\cos \theta$, where $\theta$ is the angle between the normal to the surface and the line of sight.

The model atmospheres and the angle-dependent intensities $I\left(\lambda, \theta, \varepsilon_{\mathrm{He}}, \varepsilon_{\mathrm{Si}}, \varepsilon_{\mathrm{Cr}}, \varepsilon_{\mathrm{Fe}}\right)$ mentioned above were calculated for a four-parametric grid of helium, silicon, chromium, and iron abundances (see Table 2). For silicon this grid fully covers the range of silicon abundances in the map of

\footnotetext{
${ }^{1}$ Note that the opacity caused by autoionisation is included via boundfree cross section (as default in TLUSTY).

${ }^{2}$ http://kurucz.harvard.edu
} 
Table 2. Individual abundances $\varepsilon_{\mathrm{He}}, \varepsilon_{\mathrm{Si}}, \varepsilon_{\mathrm{Cr}}$, and $\varepsilon_{\mathrm{Fe}}$ of the model grid.

\begin{tabular}{lrrrrrr}
\hline \hline $\mathrm{He}$ & -2.0 & -1.0 & & & & \\
$\mathrm{Si}$ & -4.75 & -4.25 & -3.75 & -3.25 & -2.75 & -2.25 \\
$\mathrm{Cr}$ & -6.4 & -5.9 & -5.4 & -4.9 & -4.4 & \\
$\mathrm{Fe}$ & -5.4 & -4.9 & -4.4 & -3.9 & -3.4 & \\
\hline
\end{tabular}

Kuschnig et al. (1999), but for helium, chromium, and iron the lowest abundances detected by Kuschnig et al. (1999) are omitted from the grid. Our test showed that this restriction of the grid does not influence the predicted light curves. The generation of the complete grid would require the calculation of 300 model atmospheres and synthetic spectra. Because not all abundance combinations are required for the interpolation of the Kuschnig et al. (1999) maps, we calculated only those models that were necessary. This helped us to reduce the number of calculated models by half.

\subsection{Phase-dependent flux distribution}

The radiative flux in a colour $c$ at the distance $D$ from the star with radius $R_{*}$ is (Mihalas 1978)

$$
f_{c}=\left(\frac{R_{*}}{D}\right)^{2} \int_{\substack{\text { visible } \\ \text { surface }}} I_{c}(\theta, \Omega) \cos \theta \mathrm{d} \Omega
$$

where the intensity $I_{c}(\theta, \Omega)$ at each surface point with spherical coordinates $\Omega$ is obtained by means of interpolation between the intensities $I_{c}\left(\theta, \varepsilon_{\mathrm{He}}, \varepsilon_{\mathrm{Si}}, \varepsilon_{\mathrm{Cr}}, \varepsilon_{\mathrm{Fe}}\right)$ calculated from the grid of synthetic spectra (see Table 2) as

$$
I_{c}\left(\theta, \varepsilon_{\mathrm{He}}, \varepsilon_{\mathrm{Si}}, \varepsilon_{\mathrm{Cr}}, \varepsilon_{\mathrm{Fe}}\right)=\int_{0}^{\infty} \Phi_{c}(\lambda) I\left(\lambda, \theta, \varepsilon_{\mathrm{He}}, \varepsilon_{\mathrm{Si}}, \varepsilon_{\mathrm{Cr}}, \varepsilon_{\mathrm{Fe}}\right) \mathrm{d} \lambda .
$$

The transmissivity function $\Phi_{c}(\lambda)$ of a given filter $c$ of the Strömgren photometric system is approximated for simplicity by a Gauss function (see Krtička et al. 2009, for details).

The magnitude difference is defined as

$\Delta m_{c}=-2.5 \log \left(\frac{f_{c}}{f_{c}^{\text {ref }}}\right)$,

where $f_{c}$ is calculated from Eq. (3) and $f_{c}^{\text {ref }}$ is the reference flux obtained under the condition that the mean magnitude difference over the rotational period is zero.

\section{Influence of the abundance on the emergent flux}

Individual elements modify the temperature distribution of model atmospheres by their bound-free and bound-bound transitions. This can be seen in Fig. 1, where we compare the temperature distribution of model atmospheres for typical abundances found on the surface of CU Vir. The bound-free (caused by ionisation of helium and silicon) and bound-bound transitions (line transition of chromium and iron) absorb the stellar radiation, consequently the temperature in the continuum-forming region $\left(\tau_{\text {ross }} \approx 0.1-1\right)$ increases with increasing abundance of these elements. For silicon and iron the influence of abundance on the temperature is stronger, for chromium the influence is weaker, while for typical helium abundances found on the surface of CU Vir the changes of temperature are only marginal.

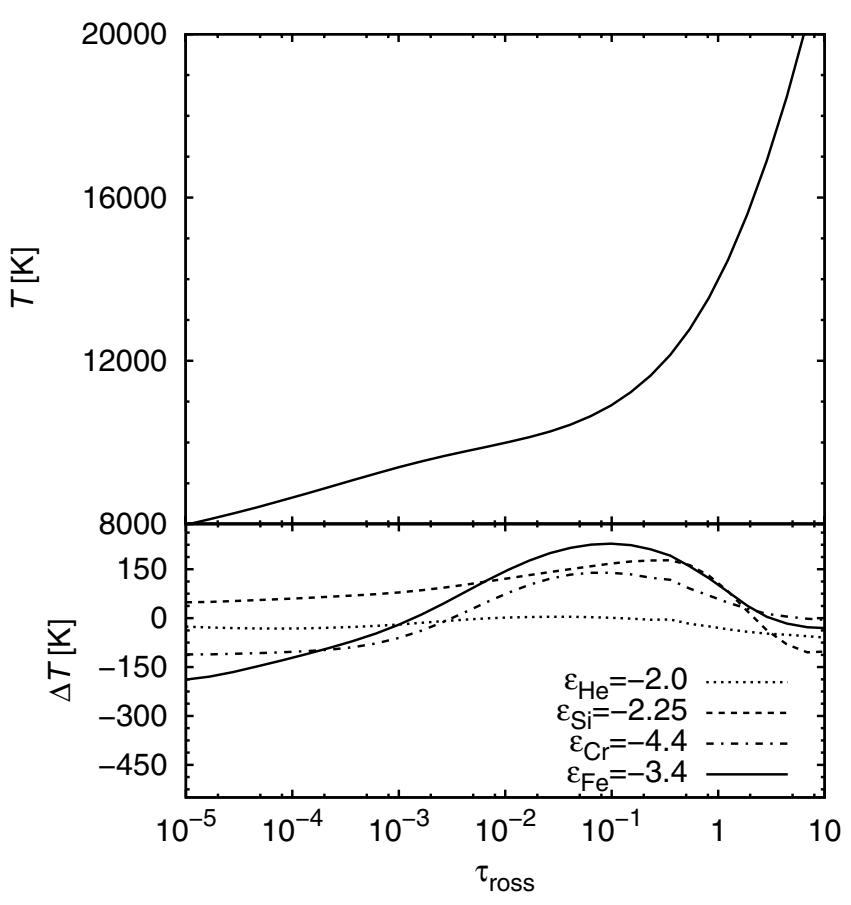

Fig. 1. Upper plot: the dependence of temperature on the Rosseland optical depth $\tau_{\text {ross }}$ in the reference model atmosphere with $\varepsilon_{\mathrm{He}}=-1.0$, $\varepsilon_{\mathrm{Si}}=-3.75, \varepsilon_{\mathrm{Cr}}=-5.9$, and $\varepsilon_{\mathrm{Fe}}=-4.4$. Lower plot: the temperature in the model atmospheres with modified abundance of individual elements minus the temperature in the reference model atmosphere.

In atmospheres with overabundant helium, silicon, chromium or iron the enhanced opacity leads to the redistribution of the flux from the short-wavelength part of the spectrum to the longer wavelengths of the UV spectrum, and also to the visible spectral regions (see Fig. 2). Consequently, the overabundant spots are bright in the uvby colours, and are dark in far-ultraviolet bands. As already found by Krtička et al. (2007), helium can affect the flux distribution only if it significantly dominates over hydrogen, i.e. for $\varepsilon_{\mathrm{He}}>0.5$. Consequently, for model atmospheres with underabundant helium the flux variations are only marginal. Note also that the flux variations caused by silicon are most pronounced in the far-UV region with $\lambda<1600 \AA$.

These flux changes can be detected as a change in the apparent magnitude. To demonstrate this, we plot (Fig. 3) the relative magnitude difference defined as

$\Delta m_{\lambda}=-2.5 \log \left(\frac{H_{\lambda}\left(\varepsilon_{\mathrm{He}}, \varepsilon_{\mathrm{Si}}, \varepsilon_{\mathrm{Cr}}, \varepsilon_{\mathrm{Fe}}\right)}{H_{\lambda}^{\mathrm{ref}}}\right)$,

against wavelength. Here $H_{\lambda}^{\mathrm{ref}}$ is the reference flux calculated for slightly overabundant chemical composition (with $\varepsilon_{\mathrm{He}}=-1.0$, $\varepsilon_{\mathrm{Si}}=-3.75, \varepsilon_{\mathrm{Cr}}=-5.9$, and $\left.\varepsilon_{\mathrm{Fe}}=-4.4\right)$. As can be seen in Fig. 3, the absolute value of the relative magnitude difference decreases with increasing wavelength. However, the behaviour of the flux calculated for modified helium is different. The maxima of the relative brightness at the positions of the hydrogen lines (especially close to the Balmer jump) remained undetected in the previous analysis. These maxima are caused by the strengthening of the Lorentz wings of the hydrogen lines owing to the higher density in the line-forming region in the models with higher helium abundance. It has not escaped our attention that the minimum of the relative magnitude difference at about 


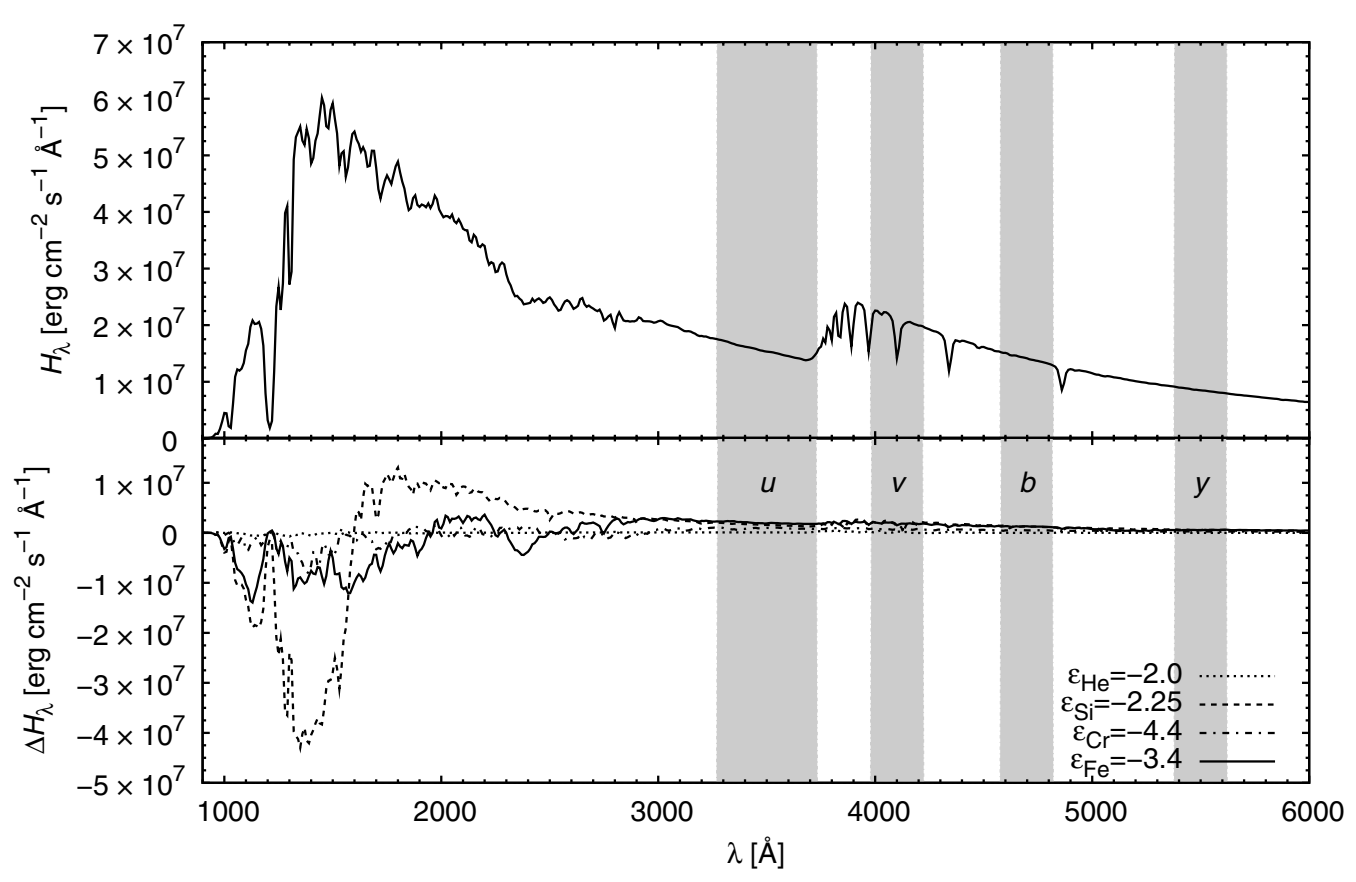

Fig. 2. Upper plot: the emergent flux from a reference model atmosphere with $\varepsilon_{\mathrm{He}}=-1.0$, $\varepsilon_{\mathrm{Si}}=-3.75, \varepsilon_{\mathrm{Cr}}=-5.9$, and $\varepsilon_{\mathrm{Fe}}=-4.4$. Lower plot: the emergent flux from the model atmospheres with modified abundance of individual elements minus the flux from a reference model. All fluxes were smoothed by a Gaussian filter with a dispersion of $10 \AA$ to show the changes in continuum, which are important for SED variability. The passbands of the uvby photometric system are also shown in the graph (grey areas).

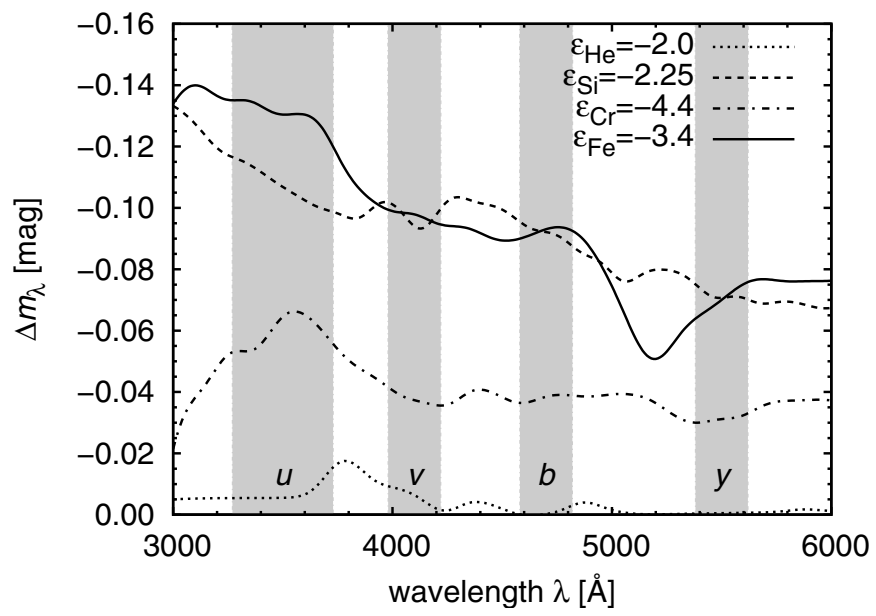

Fig. 3. Magnitude difference $\Delta m_{\lambda}$ between the emergent fluxes calculated with an enhanced abundance of individual elements and the reference flux $H_{\lambda}^{\text {ref }}$ (see Eq. (6)). The fluxes were smoothed with a Gaussian filter with a dispersion of $100 \AA$.

$5200 \AA$ caused by an accumulation of iron lines can be connected with the well-known flux depression at these wavelengths (as discussed already by, e.g., Khan \& Shulyak 2007; Krtička et al. 2009).

\section{Predicted light variations}

Predicted light curves are calculated from the surface abundance maps derived by Kuschnig et al. (1999) and from the emergent fluxes computed with the SYNSPEC code, applying Eq. (5) for individual rotational phases.

To study the influence of individual elements separately, we first calculated the light variations with the abundance map of one element only (Fig. 4), assuming a fixed abundance of other elements $\left(\varepsilon_{\mathrm{He}}=-1.0, \varepsilon_{\mathrm{Si}}=-3.75, \varepsilon_{\mathrm{Cr}}=-5.9, \varepsilon_{\mathrm{Fe}}=-4.4\right)$. From Fig. 4 it follows that iron, silicon, and chromium contribute

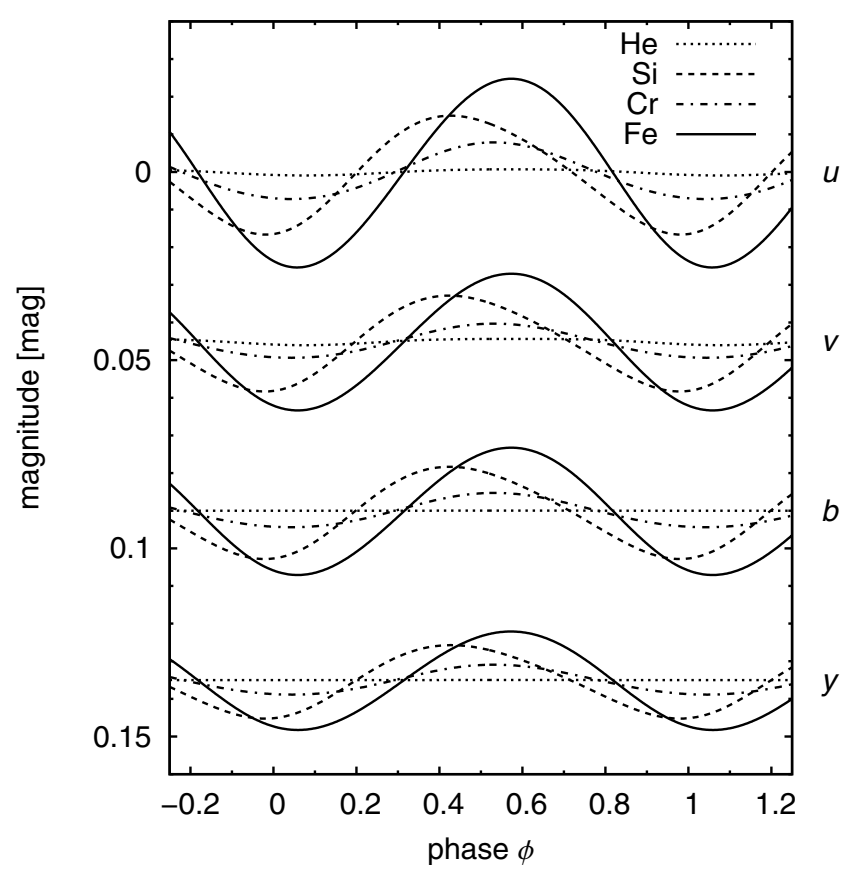

Fig. 4. Predicted light variations of CU Vir in the Strömgren photometric system calculated using abundance maps of one element only. The abundance of other elements was fixed. Light curves in individual filters were vertically shifted to better demonstrate the light variability.

most to the light variations, while the contribution of helium is only marginal. This is because of the large overabundance of these elements in the spots and by their large abundance variations on the stellar surface. The amplitude of the light variations increases with decreasing wavelength, as can be expected from the plot of the magnitude difference $\Delta m_{\lambda}$ in Fig. 3. Because the overabundant regions are brighter in the uvby colours, the predicted light variations reflect the equivalent width variations (Kuschnig et al. 1999, Fig. 1). The light maximum occurs at the 


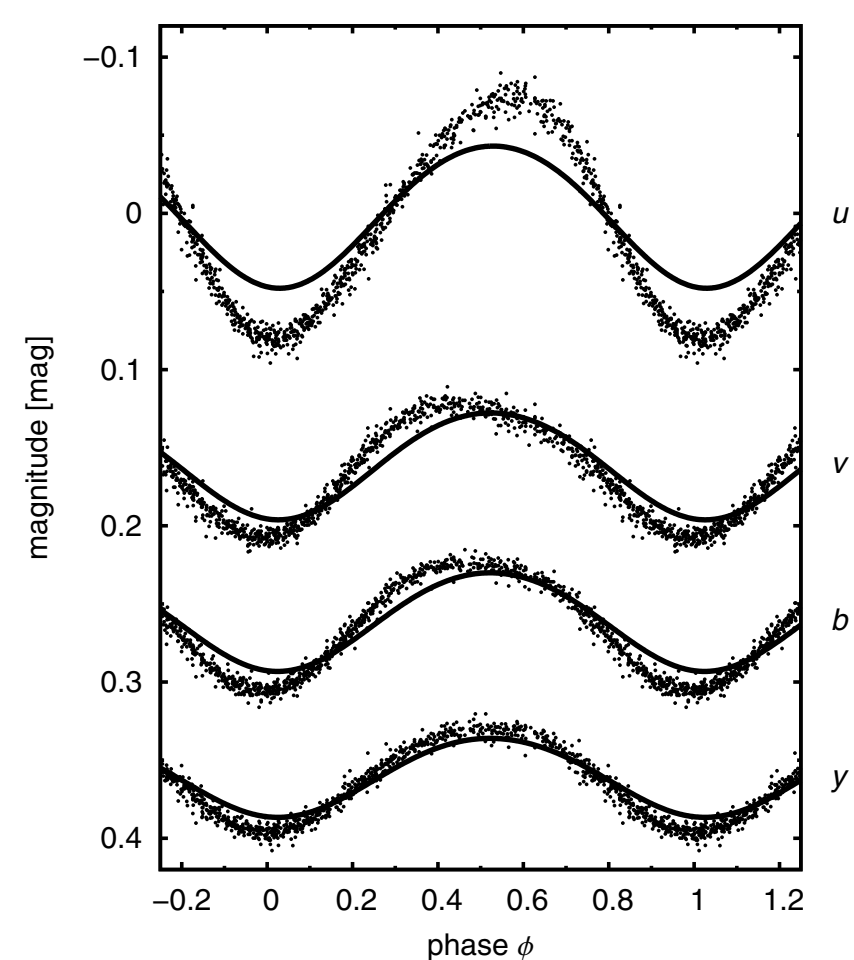

Fig. 5. Predicted light variations of CU Vir (solid lines) computed taking into account the helium, silicon, chromium, and iron surface abundance distributions derived by Kuschnig et al. (1999). The observed light variations (dots) are taken from Pyper et al. (1998). The light curves in individual filters were shifted vertically to better demonstrate the light variability.

same phase at which the equivalent widths of a given element are the largest. Because this happens at slightly different phases for individual elements, the light curves in Fig. 4 are slightly shifted.

Taking into account the surface distribution of helium, silicon, chromium, and iron in the calculation of the light curves (Fig. 5), we obtained a good agreement between the observed and predicted light curves in the $v, b$ and $y$ bands of the Strömgren photometric system. On the other hand, our models are able to explain only about half of the amplitude in the $u$ filter. The disagreement between the predicted and observed light curves is mostly apparent around phase $\phi=0.6$. Note also that a similar disagreement visible in $u$ can be also found in other filters, but to a much smaller extent. These differences clearly point to an existence of some additional, unknown mechanism working especially in the violet band that still needs to be investigated (Fig. 5, and see also Sect. 7). The discrepancies between the predicted and observed light curves increase when comparing the predicted and observed colour indices (see Fig. 6). While the $(b-y)$ data agree reasonably well, the $(v-b)$ curves are mutually shifted, and the predicted metallic index $m_{1}=(v-b)-(b-y)$ shows a significantly lower amplitude than the observed one.

The inhomogeneous surface distribution of individual elements causes bright spots on the stellar surface. The spots, whose surface distribution can be derived using abundance maps and model atmospheres (see Fig. 7), cause the light variability.

\section{Ultraviolet variations}

We have shown that the light variability of CU Vir is caused by the redistribution of flux from the far UV to the near UV and

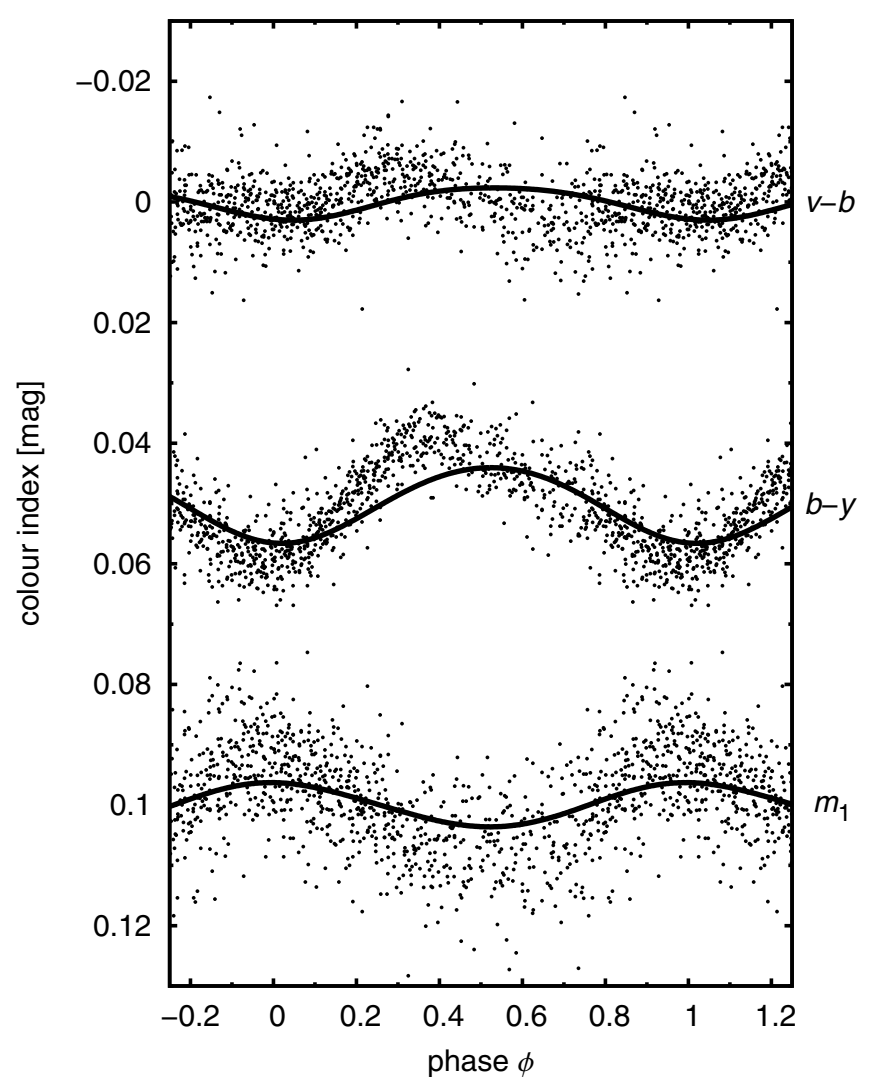

Fig. 6. Predicted variations of colour indices (solid lines) calculated from the helium, silicon, chromium, and iron surface abundance maps compared with the observations. Observed light variations (dots) are taken from Pyper et al. (1998). Light curves in individual filters were vertically shifted to better demonstrate the light variability.

visible regions. Consequently, the light variability in the far UV region should be in antiphase with the visual one. This behaviour was indeed found in a detailed analysis of IUE observations of CU Vir by Sokolov (2000).

To test these predictions quantitatively as well, we extracted IUE observations of CU Vir from the INES database (Wamsteker et al. 2000, see Table A.1) using the SPLAT package (Draper 2004; see also Škoda 2008). Here we used low-dispersion large aperture spectra in the domains 1250-1900 $\AA$ (SWP camera) and 2000-3000 ^ (LWR camera).

\subsection{Narrow-band UV variations}

As a first comparison of the UV fluxes we concentrated on narrow-band variations. For this purpose we smoothed the observed and predicted fluxes with a Gaussian filter with a dispersion of $10 \AA$.

The resulting predicted and observed UV fluxes are given in Fig. 8. To avoid possible problems with absolute IUE calibration, we normalised the predicted fluxes by a multiplicative factor, which yields the best match between observations and prediction in Fig. 8. The factor was kept fixed for all wavelengths in all subsequent calculations. Generally, the mean predicted and observed fluxes agree well, with some minor differences. The mean observed flux is slightly lower than the predicted one in the region $1600-1850 \AA$, whereas it is slightly higher in 2100-3000 $\AA$. The amplitude of the observed flux variations 

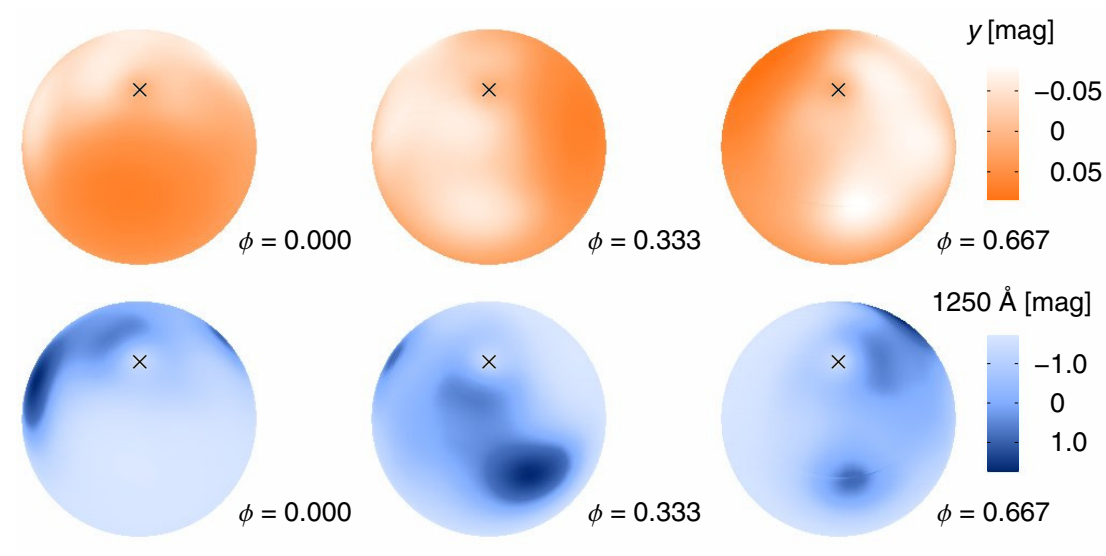

Fig. 7. Emergent intensity from individual surface elements of CU Vir at various rotational phases. Upper panel: visible $y$ band. Lower panel: UV band centred at $1250 \AA$. Both for $\mu=1$.

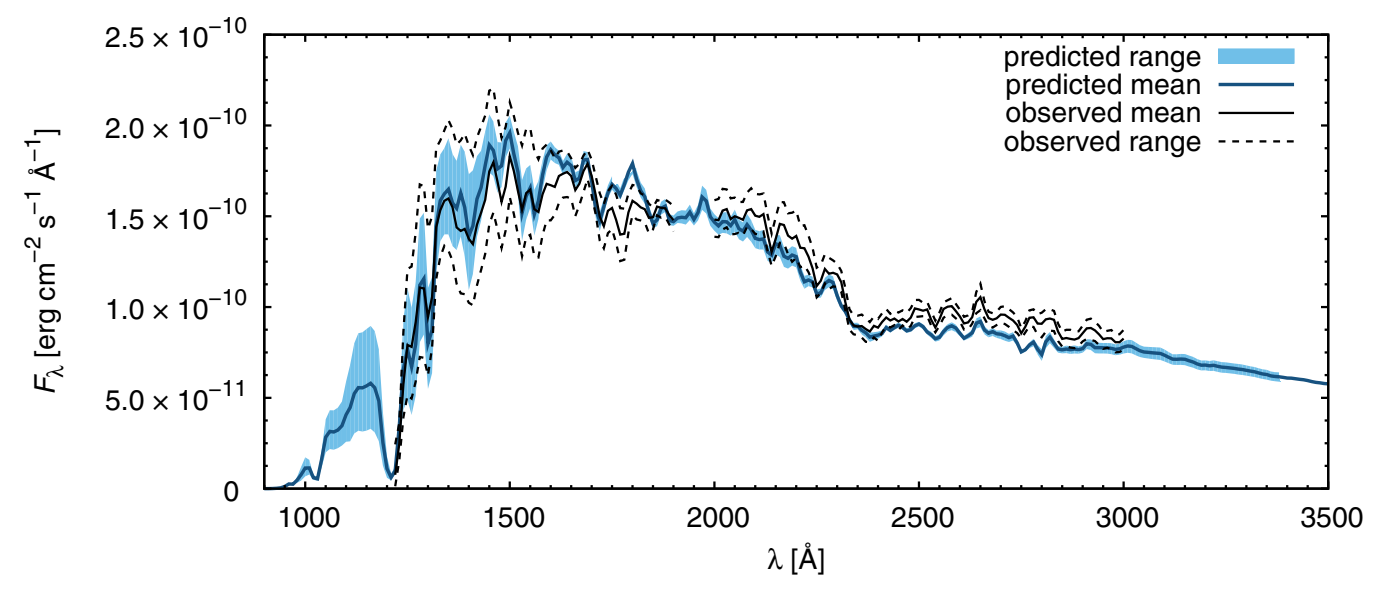

Fig. 8. Comparison of the predicted flux (mean and its variation, blue) with corresponding observed quantities (black). Both predicted and observed fluxes (IUE) were smoothed by a Gaussian filter with a dispersion of $10 \AA$. agrees well with the observed one in the regions $1250-1450 \AA$ and 2550-3000 $\AA$. In the remaining regions the predicted amplitude is lower than the observed one.

As can be seen from Fig. 9, the predicted and observed flux variations in the selected wavelengths agree very well. Our simulations are able to explain most of the observed features in the UV light curve. The amplitude is highest in the far-UV region 1250-1400 ̊, reaching nearly $1 \mathrm{mag}$ at $1250 \AA$. The light variations in this region are mainly caused by silicon (cf., Fig. 2, and Sokolov 2006, 2010). The overall agreement between the predicted and observed light curves in this region indicates that silicon abundances are well mapped.

The amplitude of the light variations is very low in the region between 1600-1900 ̊. The silicon-rich patches are dark in this wavelength region, whereas the iron-rich ones are bright, causing a near cancelation of any light variability in common. Note, however, that a fine structure of observed variations is not completely reproduced by the models, indicating either that the model atmospheres need to be improved or, which is even more likely, that the light variability has other sources.

Our models are able to nicely reproduce the observed (Sokolov 2000) antiphase variations in the far-UV on one side and the near-UV and visible region on the other side (see also Fig. 7). However, as was already clear from Fig. 8, the observed light amplitude is higher than the predicted one in the region 2000-2500 ̊. This disagreement together with the difference in the $u$ light curves (see Fig. 5) indicates a presence of an additional now unidentified source of light variability. This might be connected with a chemical element whose abundance was not mapped by Kuschnig et al. (1999). This element, together with silicon, might contribute to the light variability in this region.

Interestingly, despite the disagreement of the observed and predicted light variations in the $u$ colour of the Strömgren photometric system (Fig. 5), our models are able to nicely reproduce most of the light variations in the region 2550-3000 $\AA$ (Fig. 9). The amplitudes of the observed light curves in this region and their shapes are a result of the inhomogeneous surface distribution of silicon, iron, and chromium (see also Sokolov 2010). Even more subtle effects, like the mutual shift of the light maxima at wavelengths $2550 \AA$ and $2800 \AA$, can be explained by our models.

As can be seen from Fig. 10, the mean flux from CU Vir does not correspond to the flux calculated for the solar chemical composition. The redistribution of the flux from the far-UV to near-UV and visible regions is apparent even when comparing the average fluxes. Consequently, CU Vir is fainter than the normal stars with the same effective temperature in the far-UV on wavelengths lower than $2400 \AA$, whereas it is brighter than normal stars in the near-UV and visible regions.

\subsection{Monochromatic variations}

Although the comparison of the narrow-band variations revealed the regions where the disagreement between the observed and predicted flux variations occurs, the narrow-band variations are inadequate to figure out the origin of these variations. For this 

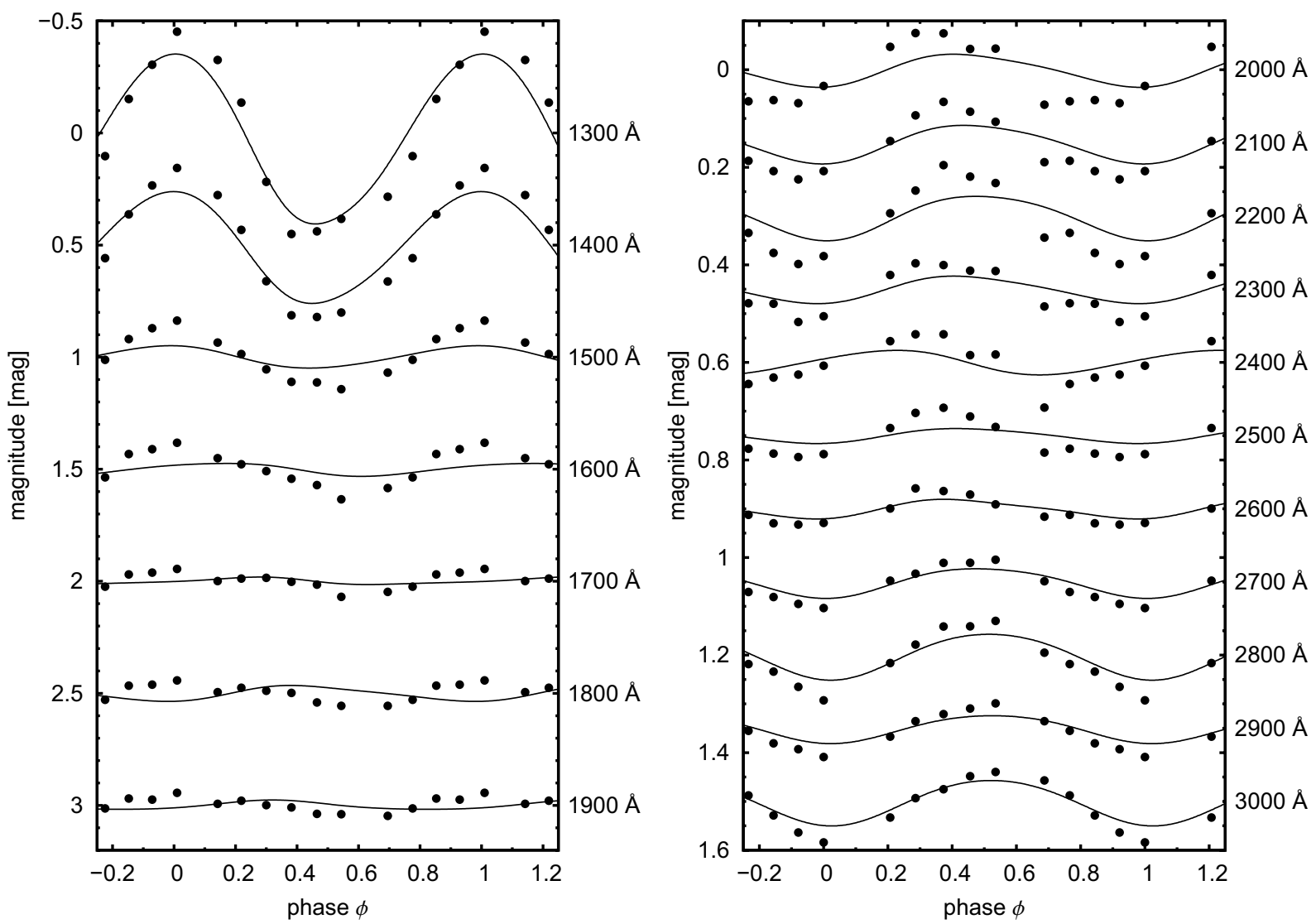

Fig. 9. Comparison of the predicted (solid line) and observed (dots) UV light variations for different wavelengths. Curves for individual wavelengths were vertically shifted to better demonstrate the variability.

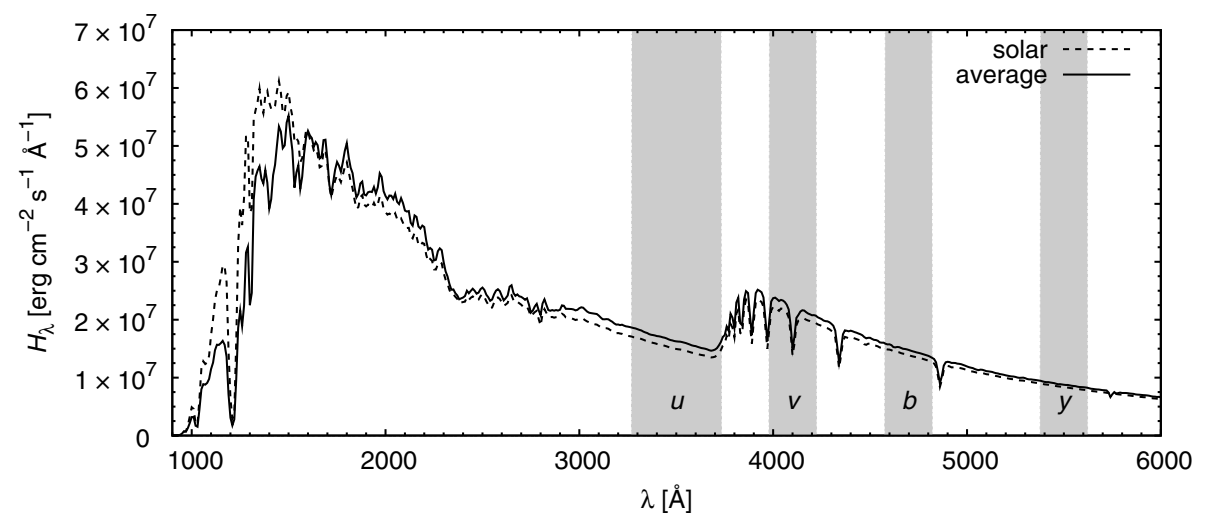

Fig. 10. Comparison of the mean predicted flux (averaged over the rotation period, solid line) and the flux roughly corresponding to the solar chemical composition $\left(\varepsilon_{\mathrm{He}}=-1.0, \varepsilon_{\mathrm{Si}}=\right.$ $-4.25, \varepsilon_{\mathrm{Cr}}=-6.4$, and $\left.\varepsilon_{\mathrm{Fe}}=-4.4\right)$. Fluxes were smoothed with a Gaussian filter with dispersion of $10 \AA$. purpose the monochromatic variations are much more convenient. To compare the monochromatic fluxes, we smoothed the predicted flux variations with a Gaussian filter with a dispersion of $1.3 \AA$, which roughly corresponds to a broadening of IUE data, and compared it with observed flux variations. The resulting monochromatic UV fluxes are given in Fig. 11.

Most features that appear as individual lines in Fig. 11 are in fact blends of a large number of individual lines (iron is a typical case). Only in a few cases it is possible to identify individual lines. The most numerous individual lines are those of silicon. The monochromatic fluxes in Fig. 11 show that even though the continuum far-UV flux in the region $\lambda<1500 \AA$ is relatively well fitted, there are some differences in the strengths of silicon lines. That the strength of the $C_{\text {II }} 1335 \AA$ doublet varies in antiphase with silicon indicates that silicon-rich regions are carbon-poor. From the weakness of the $\mathrm{Al}$ II $1671 \AA$ line we can conclude that this element is significantly underabundant with respect to the solar value.

The predicted and observed fluxes during the light minimum close to the phase $\phi=0$ agree very well. However, for phases $\phi \approx 0.5$ the observed and predicted fluxes disagree in some regions, indicating that an additional opacity source is operating here, leading to yet another redistribution of the flux from far-UV to near-UV and visible regions. From Fig. 11 it is also possible to identify these missing features that are responsible for the unexplained part of the light variability. From the plot for $\phi=0.54$ we can conclude that the missing opacity sources are located in the wavelength interval 1350-1800 ̊. 


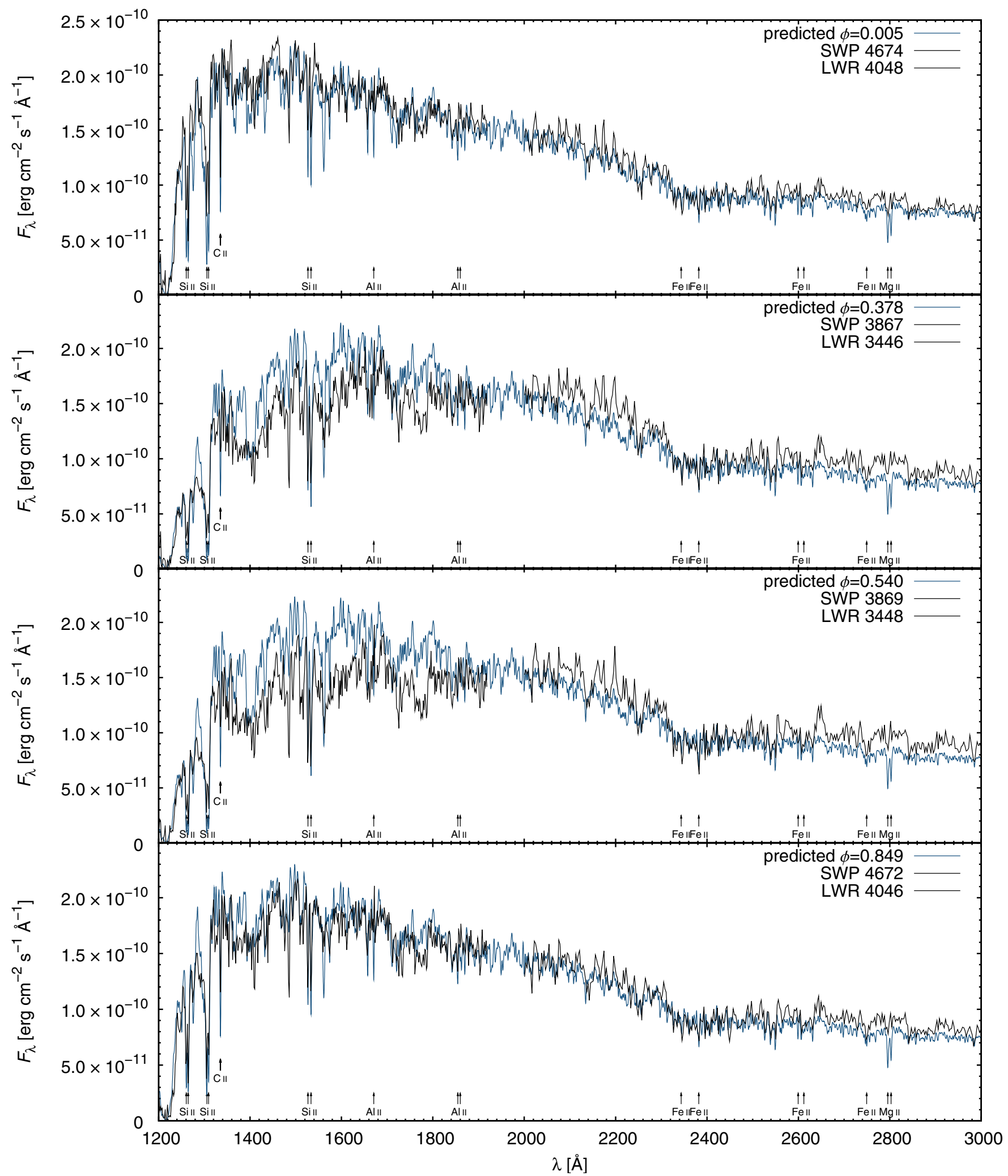

Fig. 11. Predicted and observed (IUE) flux in selected phases. Individual strong lines and iron line blends are identified.

\section{Detailed analysis of observed and simulated light curves}

\subsection{Description of observed light curves}

The detailed analysis of the observed light curves in the wavelength region from $1250 \AA$ to $7600 \AA$ was made on basis of all available photometric data of CU Vir, including very precise and reliable data obtained in 1987-1997 by Adelman et al. (1992) and Pyper et al. (1998), and the data derived from IUE spectrophotometry (see Sect. 5). The complete list of all photometric observations taken in 53 photometric bands that are quite evenly distributed along the whole spectral interval obtained in 1955-2011 is published in Mikulášek et al. (2011). For 


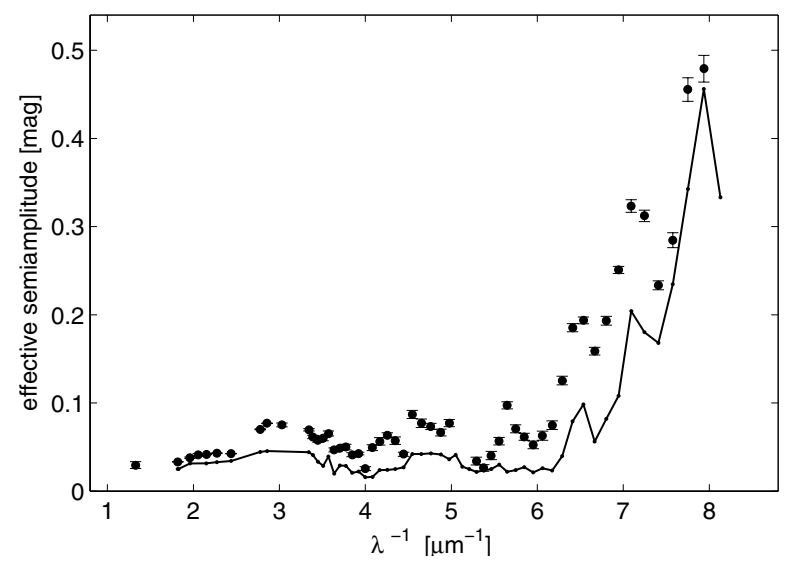

Fig. 12. Comparison of the observed effective semiamplitudes $A(\lambda)$ (see Eq. (7), dots with error bars) and semiamplitudes of simulated light variations (small dots connected by solid line).

the calculation of photometric phases we used a new ephemeris that takes into account the long-term variability of the period (see Sect. 2.1). The quantity and the quality (the mean weighted uncertainty of an individual photometric measurement is 5.3 mmag) of this photometric material enable us to investigate the photometric behaviour of CU Vir with an unprecedented accuracy.

First we studied the properties of the light curves in individual passbands. Our results show that the shapes of the light curves remained constant during the last half century. Each of them can be well expressed by a smooth single wave curve - a harmonic function of a low order. Nevertheless, the shapes of the light curves in different bands are apparently different. Applying the weighted advanced principle component analysis (APCA) to the parameters of the harmonic fits (which allows one to find hidden relationships among them, for details see e.g. a brief introduction in Mikulášek 2007) we arrived at two principal conclusions: 1) Each light curve studied can be satisfactorily well fitted by a second-order harmonic polynomial - the amplitudes of the third and higher harmonics are always bellow $0.5 \mathrm{mmag}$. 2) All light curves studied can be well expressed by a linear combination of only two basic light curves $F_{1}(\phi), F_{2}(\phi)$, the amplitude of the third and higher principal components do not exceed $1.0 \mathrm{mmag}$.

This allows us to build a relatively simple two-component phenomenological model with a minimum of free parameters valid for all studied light curves, where each of them is expressed by a linear combination of two principle functions $F_{1}(\phi), F_{2}(\phi)$ of a rotational phase $\phi$

$\Delta m(\phi, \lambda)=A(\lambda)\left[\sin \left(\frac{\pi}{2} \psi(\lambda)\right) F_{1}(\phi)+\cos \left(\frac{\pi}{2} \psi(\lambda)\right) F_{2}(\phi)\right]$,

where $A(\lambda)$ is the effective semiamplitude in the band centred on the wavelength $\lambda, \psi(\lambda)$ is the parameter explicitly determining the shape of the light curve in the wavelength $\lambda$. The functions $F_{1}(\phi), F_{2}(\phi)$ are determined by parameters $\gamma_{1}, \gamma_{2}, \phi_{11}, \phi_{12}, \phi_{21}$, and $\phi_{22}$

$$
F_{l}(\phi)=\cos \left(\gamma_{l}\right) \cos \left[2 \pi\left(\phi-\phi_{l 1}\right)\right]+\sin \left(\gamma_{l}\right) \cos \left[4 \pi\left(\phi-\phi_{l 2}\right)\right],
$$

where $l=1$ and 2 . Functions $F_{1}(\phi), F_{2}(\phi)$ were determined by means of APCA, where we confined ourselves to the first two principle components. They represent the pair of normalised mutually orthogonal vectors in the 4-D space of the Fourier coefficients. Equation (7) then means that the light curves defined by

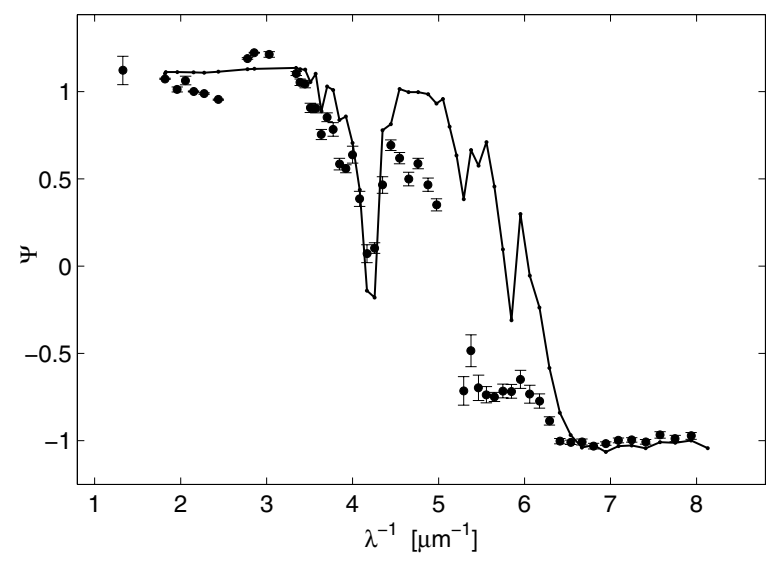

Fig. 13. Comparison of the form of observed light curves described by the dimensionless value $\psi$ (see Eq. (7), dots with error bars) and with the value predicted by our models (small dots connected by solid line).

their vector in the space of the Fourier coefficients should lay in the plane determined by these two vectors of $F_{1}(\phi), F_{2}(\phi)$ functions.

The light curves reach the maximum amplitude for wavelengths shorter than $1600 \AA$, whereas their shapes are nearly identical in this region (see Figs. 12, 13, and Table A.2). That is why we rotated the orthogonal vector base in its plane by an angle of -0.12 rad so that the $x$-axis intersects these points. The parameters of the principle light curves $F_{1}(\phi), F_{2}(\phi)$ are then $\gamma_{1}=$ $0.1244 \mathrm{rad}, \phi_{11}=-0.0132, \phi_{12}=0.00442, \gamma_{2}=0.2468 \mathrm{rad}$, $\phi_{21}=-0.2655$, and $\phi_{22}=0.0912$.

\subsection{Comparison of observed and predicted light curves}

The shapes of the simulated light curves can be described by the same procedure as the observed ones, consequently we can compare them unambiguously. We have arrived at the following conclusions:

1. The shapes of both the observed and the simulated light curves strongly depend on the wavelength. There are differences not only in the amplitudes of variations $A(\lambda)$ but also in their shapes, quantified by the parameter $\psi(\lambda)$. This can be understood as the result of the influence of several elements (at least $\mathrm{Si}, \mathrm{Fe}, \mathrm{Cr}$ ).

2. The shapes of the basic phase curves $F_{1}(\phi), F_{2}(\phi)$ and the dependence of their parameters $A(\lambda)$ and $\psi(\lambda)$ on wavelength can be caused by two vast overlapping photometric spots centred at phases $\phi_{01}=0.3209(24)$ and $\phi_{02}=0.6244(23)$ with different contrasts on the stellar surface. These spots can be identified as spots with overabundant $\mathrm{Si}$ and iron-peak elements $(\mathrm{Cr}, \mathrm{Fe})$.

3. The uneven colours of the photometric spots described by the parameter $\psi$ are illustrated in Fig. 9 with the IUE light curves in wavelengths $1500 \AA$, $2100 \AA$, and $3000 \AA$.

4. The dissimilar spectral energy distribution in spots is the reason why we do not find any 'null' point with zero amplitude of the flux variations.

5. The simulation of the light variations cannot fully explain the observed amplitudes in the whole studied spectral region. The simulated amplitudes are on average smaller by a few tens of percent than the observed ones (see Fig. 12), which implies that we have neglected some of the important sources of the light variations in our modelling. 


\section{Discussion}

Despite the overall good agreement between the predicted and observed light curves, there still remain some differences between the simulations and observations. These are especially apparent in the Strömgren $u$ filter and in the UV region 2000-2500 ̊. Note, however, that CU Vir has the highest amplitude of all stars modelled in detail so far, consequently it is encouraging that even this high amplitude can be explained for the most part. Here we discuss some possible reasons for the remaining disagreements.

\subsection{Limitations of abundance maps}

The limitations of abundance maps used could be an important source of discrepancy between prediction and observation. While the fine structure of abundance maps does not significantly influence the predicted variability (as shown by Krtička et al. 2009 using different abundance maps of Lehmann et al. 2007), other effects may be significant. The predicted SED variations are sensitive to the maximum abundance and abundance amplitude of a given element in the map. The observed variations of silicon equivalent widths are nicely reproduced by the abundance maps (see Fig. 1 of Kuschnig et al. 1999), indicating their high reliability. This is also supported by a good agreement between predicted and observed SED variations in the far-UV region, where the silicon dominates. On the other hand, the predicted and observed equivalent widths of chromium and iron in Fig. 1 of Kuschnig et al. (1999) disagree to some extent. Our tests showed that especially the modified abundance of iron could help to explain some part of the discrepancy between theory and observations.

Another source of the discrepancies might be connected with limitations of the model atmospheres used for the abundance analysis. All these considerations point to a need of new more precise abundance maps of CU Vir.

\subsection{Influence of additional elements}

We have shown that most of the light variability of CU Vir is caused by the uneven distribution of chemical elements. Consequently, it is possible that a part of the remaining discrepancy between theory and observation is also caused by other element(s), whose surface distribution was not mapped by Kuschnig et al. (1999). Both the optical and UV light curves provide strong constraints on the opacity caused by this as yet unidentified element, which might redistribute the flux from UV region of about 2000-2500 $\AA$, especially to the region of Strömgren $u$.

We tested if either of the chemical elements currently included in the TLUSTY model atmospheres could cause these light variations. Excluding magnesium because of its low abundance previously, no other element included in TLUSTY (i.e., $\mathrm{C}, \mathrm{N}, \mathrm{O}, \mathrm{Ne}, \mathrm{Al}$, and $\mathrm{S}$ ) is able to cause the remaining light variations observed in CU Vir. Consequently, it is likely that another element (especially the iron-peak ones) could be the cause.

Very recent observations in a broad spectral range suggest that titanium and oxygen could also be contributors to inhomogeneous abundance structures on the surface of CU Vir. Especially titanium (provided it is significantly overabundant) is one of the potential causes of the UV variations we cannot simulate so far.

\subsection{Vertical abundance stratification}

Vertical abundance stratification is observed in some CP stars (e.g., Ryabchikova 2004). Sokolov (2010) proposed that the vertical abundance stratification may influence the light variability. Our test calculations confirmed these expectations. The models with overabundant iron in the outer regions (for the Rosseland optical depth $\left.\tau_{\text {Ross }}<0.1\right)$ indeed show a larger magnitude difference in $u$ than in the $v, b$, and $y$ colours. Consequently, vertical abundance stratification could possibly explain the difference between the phases of maxima in individual Strömgren filters. However, the influence of vertical abundance stratification on the UV region is relatively low, consequently another process is needed to explain the difference between observation and theory in this region.

\subsection{Surface temperature variations}

Surface temperature differences and variable temperature gradients were also suggested as possible causes of the CP star light variability (Weiss et al. 1976; Stępień 1978). Hot stars may retain subsurface convection zones (Cantiello et al. 2010), which can generate local magnetic fields. These magnetic fields may give rise to the surface temperature differences, and consequently cause the light variability. However, mild differences between the observed and predicted light curves do not indicate any (effective) temperature differences on the surface of CU Vir. This is supported also by the fact that e. g. the predicted mean of the flux distribution simulates the observed one fairly well, as depicted in Fig. 8. Moreover, a good agreement between the observed and predicted light curves in the far-UV region and the $v b y$ light curves in the visible region excludes the temperature differences as a main source of the light variability.

\subsection{Influence of the turbulent velocity}

In our study we assumed a generic value of the microturbulent velocity $2 \mathrm{~km} \mathrm{~s}^{-1}$. This parameter, which roughly accounts for atmospheric velocity fields, likely has a zero value in corresponding normal stars (Landstreet et al. 2009). We kept a nonzero value here as a very rough approximation of Zeeman line broadening. The adopted value of the microturbulent velocity may, however, influence the emergent flux. At higher microturbulent velocities the line transitions are able to absorb radiation more effectively, increasing thus the temperature in the continuum forming regions. To estimate the magnitude of this effect, we calculated an additional model with higher microturbulent velocity $4 \mathrm{~km} \mathrm{~s}^{-1}$ and assuming enhanced abundance of heavier elements $\left(\varepsilon_{\mathrm{He}}=-1.0, \varepsilon_{\mathrm{Si}}=-2.25, \varepsilon_{\mathrm{Cr}}=-4.9\right.$, $\left.\varepsilon_{\mathrm{Fe}}=-3.4\right)$. We compared the resulting flux distribution with the model with the same chemical composition, but with a standard microturbulent velocity of $2 \mathrm{~km} \mathrm{~s}^{-1}$. The calculated magnitude difference (Eq. (5)) between these models has its minimum $-0.05 \mathrm{mag}$ in the near-UV region $3000-3800 \AA$ and a maximum about -0.10 mag in the region $2200-2550 \AA$. These are the regions where the most apparent differences between observed and predicted light variations occur. Consequently, a higher value of the microturbulent velocity and/or surface microturbulent velocity distribution (see Sect. 7.8) cannot be ruled out as a possible cause of the remaining difference between theory and observation.

\subsection{The effects of fast rotation}

CU Vir belongs to the fast rotators among CP stars. From Table 1 we can infer its rotational velocity $v_{\text {rot }}=320 \mathrm{~km} \mathrm{~s}^{-1}$, indicating 
a rotational velocity close to the critical one. To quantify this, the stellar radius has to be known with sufficiently high precision. Using the stellar parameters derived from spectroscopy and photometry (i.e., $v_{\text {rot }} \sin i$, inclination, and period, see Table 1), we can estimate the equatorial radius to be $R_{\mathrm{eq}}=3.3 \pm 0.6 R_{\odot}$. This value agrees well with the stellar radius derived from the evolutionary tracks in the $T_{\text {eff }}-\log g$ plane of Schaller et al. (1992), which is $R=3.2 \pm 0.5 R_{\odot}$ (the derived mass is $M=$ $\left.3.8 \pm 0.2 M_{\odot}\right)$. Note, however, that this yields a significantly higher radius than that derived from the observed UV flux, which is $R=1.9 \pm 0.1 R_{\odot}$ assuming a distance of $79 \pm 1 \mathrm{pc}$ (van Leeuwen 2007). This possibly points either to a problem with the absolute flux calibration, or to an inclination that is too low. The latter is supported by a lower radius of $R=2.3 \pm 0.1 R_{\odot}$ derived from photometry and evolutionary tracks by Kochukhov \& Bagnulo (2006).

To study the effect of fast rotation at its extremum, we assumed the equatorial radius $R_{\mathrm{eq}}=3.3 R_{\odot}$, and $M=3.8 M_{\odot}$, the polar radius is then $R_{\mathrm{p}}=R_{\mathrm{eq}}\left(1+v_{\mathrm{rot}}^{2} R_{\mathrm{eq}} /(2 G M)\right)^{-1}=2.7 R_{\odot}$ (Collins 1963). The ratio of the rotational velocity to the critical one is then $v_{\text {rot }} / v_{\text {krit }}=v_{\text {rot }} / \sqrt{2 G M / 3 R_{\mathrm{p}}}=0.75$. If the star rotates this rapidly, the polar to equator difference in the effective surface gravity and effective temperature are $\Delta \log g=$ $\log \left(G M / R_{\mathrm{p}}^{2}\right)-\log \left(G M / R_{\mathrm{eq}}^{2}-v_{\text {rot }}^{2} / R_{\mathrm{eq}}\right) \approx-0.4$ and $\Delta T_{\text {eff }} \approx$ $3000 \mathrm{~K}$ (assuming $T_{\text {eff }}(\vartheta) \sim g^{1 / 4}(\vartheta)$, von Zeipel 1924; Slettebak 1949; Owocki et al. 1994).

The variations of local surface gravity and effective temperature connected with a fast rotation by itself cannot raise any light variability (assuming fixed axis of rotation) because of their axial symmetry. However, even axisymmetric surface variations may modify the light variations caused by inhomogeneous elemental surface distribution via several effects. First, surface layers with different abundances may respond differently to the temperature and surface gravity variations, modifying the flux distribution. Second, the area of the surface element is modified through the oblateness of the surface of a rotating star. Finally, the direction of the beam pointing to the observer relative to the local outward normal and its cross-section are different on spherical and oblate surface.

To test the influence of these effects, we calculated an additional grid of model atmospheres corresponding to the stellar equator $\left(T_{\text {eff,eq }}=11500 \mathrm{~K}, \log g_{\text {eq }}=3.71\right)$ and to the pole $\left(T_{\text {eff, }}=14300 \mathrm{~K}, \log g_{\mathrm{p}}=4.16\right)$ with different abundances of silicon and iron (after Table 2). The polar and equatorial effective temperature and gravity were calculated assuming that the CU Vir parameters $T_{\text {eff }}, g$ derived by Kuschnig et al. (1999) represent some kind of mean over the stellar surface and that these parameters correspond to some particular surface region. In this case $T_{\text {eff }}(\vartheta)=[g(\vartheta) / g]^{1 / 4} T_{\text {eff. }}$. For simplicity we assumed a fixed abundance of helium and chromium here $\left(\varepsilon_{\mathrm{He}}=-1, \varepsilon_{\mathrm{Cr}}=5.9\right)$ because these elements are not the main sources of the light variability. The resulting light curve was derived using Eq. (5), now interpolating the intensities in Eq. (4) also between the models corresponding to the pole and to the equator.

The inclusion of the gravity darkening only (with the latitude-dependent radius calculated after Harrington \& Collins 1968) leads to the decrease of predicted amplitude of light variations by up to $0.01 \mathrm{mag}$. This is because for both silicon and iron the values of $\Delta m_{\lambda}$ Eq. (5) decrease with decreasing temperature.

The inclusion of different surface areas caused by the oblateness of the star leads to another small modification of the light curve, which is mostly negligible (the difference is up to one millimagnitude). We did not consider the effect of the difference of beams pointing to the observer (in an oblate star compared to the spherical one), but given the small influence of other effects, we expect that this is also negligible.

We conclude that the effect of the gravity darkening modifies the light curves, but it does not seem to be the main reason for the discrepancy between predicted and observed light curves. But the effect of gravity darkening on the light curves introduces a new possibility to test the theory of gravity darkening in the future. To this end, the influence of this effect on the abundances derived from the Doppler imaging has also to be accounted for.

\subsection{Influence of the magnetic field}

$\mathrm{CU}$ Vir is known to host a large-scale magnetic field, which could, in principle, contribute to the light variability via the rotational modulation of the magnetic field intensity and thus opacity in Zeeman broadened spectral features. Assuming a dipolar field geometry Trigilio et al. (2000) based on phase-resolved longitudinal magnetic field measurements from Borra \& Landstreet (1980) determined a polar magnetic field $B_{\mathrm{p}}=3 \mathrm{kG}$ and the angle between the stellar rotation axis and the line-of-sight $\beta=74^{\circ}$. Regarding magnetic CP stars, this is a fairly moderate magnetic field and, as demonstrated by Khan \& Shulyak (2006) based on detailed model atmosphere calculations with anomalous Zeeman effect and polarized radiative transfer, such a field does not produce significant effects on parameters observed in different photometric systems. In addition, with the inclination angle $i=30^{\circ}$ the surface average magnetic field modulus varies only by $\Delta\langle|\boldsymbol{B}|\rangle \approx 300 \mathrm{G}$ during the rotational cycle, which is far too small to induce any detectable changes in light-curves.

On the other hand, as shown by Shulyak et al. (2008), the combined impact of the magnetic field and the realistic chemistry could be more important than the effect of using only individual abundances. Thus, the effect of including the magnetic field in the computations of photometric parameters would be more stronger in regions of spots with enhanced abundances compared to the case of a homogeneously stratified atmosphere.

Because we are mostly interested in the estimate of the maximum possible amplitude that the magnetic field could introduce in the light curves relative to the inhomogeneously distributed abundances, we computed several magnetic model atmospheres using the LLmodels code (Shulyak et al. 2004). Anomalous Zeeman splitting and polarised radiative transfer were included as described in Khan \& Shulyak (2006). We found that the highest impact of the magnetic field for a spot with enhanced Fe, $\mathrm{Si}$, and $\mathrm{Cr}$, located at the pole with $|\boldsymbol{B}|=3 \mathrm{kG}$ and equator with $|\boldsymbol{B}|=1.5 \mathrm{kG}$ amounts to $\Delta u \approx 0.005 \mathrm{mag}$. This difference subsequently decreases for other Strömgren filters. Accounting for the surface averaged magnetic field modulus results in $\Delta\langle|\boldsymbol{B}|\rangle=2.2 \mathrm{kG}$ at the phase when the magnetic pole is visible and $\Delta\langle|\boldsymbol{B}|\rangle=1.9 \mathrm{kG}$ at the phase of magnetic equator respectively. The effect is then reduced to $\Delta u \approx 0.001 \mathrm{mag}$. All this is of an order of magnitude less than needed to explain the deviations between observed and predicted non-magnetic amplitudes of the $u$-parameter shown in Fig. 5. We therefore conclude that the magnetic field has little or negligible effect on the lightcurve appearance and can be ignored in the present study.

\subsection{Convection zone in helium-rich models}

In addition to the central convection zone, a hot star may have subsurface iron and helium convective zones (Maeder 1980; Maeder et al. 2008; Cantiello et al. 2010). Our models show that 
for helium-rich models with $\varepsilon_{\mathrm{He}} \gtrsim 0$, the helium convection zone moves towards the stellar surface and its top is located in the model atmosphere for Rosseland optical depths $\tau_{\text {Ross }} \approx 1-100$. The existence of a subsurface convection zone may have interesting astrophysical consequences (e.g., Maeder et al. 2008; Cantiello et al. 2010). The convective zone may create a dynamo, generating the chromospheric activity and consequently also Xray emission, whose existence is still puzzling in A type stars (Schröder \& Schmitt 2007). Moreover, convection may cause surface turbulence, leading to inhomogeneous distribution of the turbulent velocity provided helium is also distributed inhomogeneously.

However, these effects are likely strongly damped in CU Vir, which has a strong surface magnetic field (Landstreet \& Borra 1977). Our results show that the magnetic field energy density dominates over the gas energy density to the optical depths of about $\tau_{\text {Ross }} \approx 10^{3}$, suppressing any motion perpendicular to the magnetic field lines.

\subsection{NLTE effects}

Although the TLUSTY model atmosphere code enables us to calculate NLTE models, we confined ourselves to LTE models, because we expect NLTE effects to be marginal for the light variability. To test this we calculated additional NLTE models. Because we do not have sufficient atomic data to calculate NLTE models including chromium, we forced LTE for this element, even if for the remaining elements we assumed NLTE.

We calculated an NLTE model with enhanced abundance of heavier elements $\left(\varepsilon_{\mathrm{He}}=-1.0, \varepsilon_{\mathrm{Si}}=-2.25, \varepsilon_{\mathrm{Cr}}=-4.9\right.$, $\left.\varepsilon_{\mathrm{Fe}}=-3.4\right)$ and compared it with the corresponding LTE model. The resulting fluxes differ by about $1-2 \%$ in the visible and near-UV regions. The most pronounced changes appear in the far-UV region with $\lambda<1500 \AA$. Consequently, the changes owing to NLTE are significantly lower than those owing to variable abundances, and cannot be the main source of the difference between theory and observations.

The NLTE effects are, however, significant in lines. The NLTE effects lead not only to the well-known strengthening of the core of hydrogen lines (e.g., Lanz \& Hubeny 2007), but the line equivalent widths of other elements are also slightly affected. Finally, in NLTE some infrared lines appear in emission.

\section{Conclusions}

We successfully simulated the UV and visual SED variability of the helium-weak star CU Vir. We assumed that the light variability is caused by the inhomogeneous surface distribution of elements and used model atmospheres to predict the light variability.

Individual chemical elements are distributed inhomogeneously on the surface of CU Vir, as derived by Kuschnig et al. (1999) by Doppler mapping. The chemical composition influences the emergent flux through flux redistribution from the farUV to near-UV and visible regions. The bound-free transitions of silicon and the bound-bound transitions of iron and chromium mostly cause the flux redistribution in CU Vir. As a result of the flux redistribution, the individual surface elements display different brightness in individual photometric bands, although the total (frequency integrated) emergent flux is the same for all surface elements. The inhomogeneous surface brightness manifests itself by the light variations caused by stellar rotation.
The inhomogeneous surface distribution of silicon, chromium, and iron is able to explain most of the observed UV and visible SED variations. We successfully reproduced the antiphase behaviour of the light curves in the far-UV and visible regions. We emphasise that the variability seen in the visible is just a faint gleam of the variability seen in the UV. While the amplitude of the light curves merely reaches about a few hundredths of magnitude in the visual domain, it reaches about $1 \mathrm{mag}$ in the UV. However, our models are able to reproduce just part of the variability seen in the UV region 2000-2500 $\AA$ by IUE and in the $u$ filter of the Strömgren photometric system. Another mechanism(s) has to be invoked to explain this difference between the observed and predicted light curves. This so far unidentified mechanism could be connected with inhomogeneous distribution of an element whose surface abundance distribution was not mapped by Kuschnig et al. (1999), but also other effects may contribute to this difference.

Our models nicely reproduce the observed SED for the phase $\phi \approx 0$, when the regions with lowest elemental abundances are seen. On the other hand, there are some discrepancies between simulated and observed SED for the phase $\phi \approx 0.5$, when the overabundant regions appear. These discrepancies have likely the same origin as the differences of the light curves.

Our study provides additional evidence that the light variability of chemically peculiar stars is mostly caused by the inhomogeneous surface distribution of individual chemical elements, flux redistribution, and stellar rotation. The comparison of observed and predicted SED and its variation may serve as a test of opacity sources included in current model atmospheres. Finally, as a byproduct, it provides an image of the stellar surface, which (besides the interferometry) is the only way how these images can be derived.

Acknowledgements. We wish to thank Dr. P. Škoda for his continuous and enthusiastic work on the Virtual Observatory project. This work was supported by grants GAAV IAA301630901, MEB 061014/WTZ CZ 10-2010, Deutsche Forschungsgemeinschaft (DFG) Research Grant RE1664/7-1 to DS, and VEGA 2/0074/09. This research was partly based on the IUE data derived from the INES database using the SPLAT package. This research made use of NASA's Astrophysics Data System, the SIMBAD database, operated at the CDS, Strasbourg, France and the on-line database of photometric observations of mCP stars (Mikulášek et al. 2007). The access to the METACentrum (super)computing facilities provided under the research intent MSM6383917201 is also acknowledged.

\section{References}

Adelman, S. J., Dukes, R. J. Jr., \& Pyper, D. M. 1992, AJ, 104, 314

Asplund, M., Grevesse, N., \& Sauval, A. J. 2005, Cosmic Abundances as Records of Stellar Evolution and Nucleosynthesis, ed. T. G. Barnes III, F. N. Bash (San Francisco: ASP), ASP Conf. Ser., 336, 25

Bautista, M. A. 1996, A\&AS, 119, 105

Bautista, M. A., \& Pradhan, A. K. 1997, A\&AS, 126, 365

Bohlender, D. A., Rice, J. B., \& Hechler, P. 2010, A\&A, 520, A44

Borra, E. F., \& Landstreet, J. D., 1980, ApJS, 42, 421

Butler, K., Mendoza, C., \& Zeippen, C. J. 1993, J. Phys. B, 26, 4409

Cantiello, M., Langer, N., Brott, I., et al. 2009, A\&A, 499, 279

Collins, G. W. 1963, ApJ, 138, 1134

Draper, P. W. 2004, SPLAT: A Spectral Analysis Tool, Starlink User Note, 243 (University of Durham)

Fernley, J. A., Hibbert, A., Kingston, A. E., \& Seaton, M. J. 1999, J. Phys. B, 32, 5507

Harrington, J. P., \& Collins, G. W. 1968, ApJ, 151, 1051

Hardie, R. 1956, AJ, 61, 178

Hibbert, A., \& Scott, M. P. 1994, J. Phys. B, 27, 1315

Hubeny, I. 1988, Comput. Phys. Commun., 52, 103

Hubeny, I., \& Lanz, T. 1992, A\&A, 262, 501

Hubeny, I., \& Lanz, T. 1995, ApJ, 439, 875

Kellett, B. J., Graffagnino, V., Bingham, R., Muxlow, T. W. B., \& Gunn, G. A. 2007 [arXiv: astro-ph/0701214v1] 
Khan, S. A. \& Shulyak, D. 2006, A\&A, 448, 1153

Khan, S. A., \& Shulyak, D. 2007, A\&A, 469, 1083

Khokhlova, V.L., Vasilchenko, D. V., Stepanov, V. V., \& Romanyuk, I. I. 2000, AstL, 26, 177

Kochukhov, O., \& Bagnulo, S. 2006, A\&A, 450, 763

Kochukhov, O., Khan, S., \& Shulyak, D. 2005, A\&A, 433, 671

Kodaira, K. 1973, A\&A, 26, 385

Krivosheina, A. A., Ryabchikova, T. A., \& Khokhlova, V.L. 1980, Nauchnye Informatsii, Ser. Astrof., 43, 70

Krtička, J., Mikulášek, Z., Zverko, J., \& Zižňovský, J. 2007, A\&A, 470, 1089

Krtička, J., Mikulášek, Z., Henry, G. W., et al. 2009, A\&A, 499, 567

Kurucz, R. L. 1994, Kurucz CD-ROM 22, Atomic Data for Fe and $\mathrm{Ni}$ (Cambridge: SAO)

Kuschnig, R., Ryabchikova, T. A., Piskunov, N. E., Weiss, W. W., \& Gelbmann, M. J. 1999, A\&A, 348, 924

Landstreet, J. D., \& Borra, E. F. 1977, ApJL, 212, 43

Landstreet, J. D., \& Borra, E. F. 1978, ApJL, 224, 5

Landstreet, J. D., Kupka, F., Ford, H. A. et al. 2009, A\&A, 503, 973

Lanz, T., \& Hubeny, I. 2003, ApJS, 146, 417

Lanz, T., \& Hubeny, I. 2007, ApJS, 169, 83

Lanz, T., Artru, M.-C., Le Dourneuf, M., \& Hubeny, I. 1996, A\&A, 309, 218

Lehmann, H., Tkachenko, A., Fraga, L., Tsymbal, V., \& Mkrtichian, D. E. 2007, A\&A, 471, 941

Lüftinger, T., Kochukhov, O., Ryabchikova, T., et al. 2010, A\&A, 509, A71

Luo, D., \& Pradhan, A. K., 1989, J. Phys. B, 22, 3377

Maeder, A. 1980, A\&A, 90, 311

Maeder, A., Georgy, C., \& Meynet, G. 2008, A\&AL, 479, 37

Mendoza, C., Eissner, W., Le Dourneuf, M., \& Zeippen, C. J. 1995, J. Phys. B, 28,3485

Michaud, G. 2004, in The A-Star Puzzle, ed. J. Zverko, J. Žižňovský, S. J. Adelman, \& W. W. Weiss (Cambridge: Cambridge Univ. Press), IAU Symp., 224, 173

Mihalas D. 1978, Stellar Atmospheres (San Francisco: Freeman \& Co., 1978)

Mikulášek, Z. 2007, Astron. Astrophys. Transact. 26, 63

Mikulášek, Z., Janík, J., Zverko, J., et al. 2007, Astron. Nachr., 328, 10

Mikulášek, Z., Krtička, J., Henry, G. W., et al. 2011, A\&A, 534, L5

Molnar, M. R. 1973, ApJ, 179, 527

Nahar, S. N. 1996, Phys. Rev. A, 53, 1545

Nahar, S. N. 1997, Phys. Rev. A, 55, 1980

Nahar, S. N., \& Pradhan, A. K. 1993, J. Phys. B 26, 1109

Nakajima, R. 1985, Ap\&SS, 116, 285

Owocki, S. P., Cranmer, S. R., \& Blondin, J. M. 1994, ApJ, 424, 887

Peach, G., Saraph, H. E., \& Seaton, M. J. 1988, J. Phys. B, 21, 3669

Peterson, D. M. 1970, ApJ, 161, 685

Piskunov, N., \& Kochukhov, O. 2002, A\&A, 381, 736

Pyper, D. M., \& Adelman, S. J. 2004, in The A-Star Puzzle, ed. J. Zverko, J. Žižňovský, S. J. Adelman, \& W. W. Weiss (Cambridge: Cambridge Univ. Press), IAU Symp., 224, 307

Pyper, D. M., Ryabchikova, T., Malanushenko, V., et al. 1998, A\&A, 339, 822

Rice, J. B., Wehlau, W. H., \& Khokhlova, V. L. 1989, A\&A, 208, 179

Ryabchikova, T. A. 1991, in The Sun and Cool Stars. Activity, Magnetism, Dynamos, IAU Colloq. 130, ed. I. Tuominen, D. Moss, \& G. Rüdiger (Berlian: Springer Verlag), Lecture Notes in Physics, 380, 350

Ryabchikova, T. 2004, in The A-Star Puzzle, ed. J. Zverko, J. Žižňovský, S. J. Adelman, \& W. W. Weiss (Cambridge: Cambridge Univ. Press), IAU Symp., 224,283

Schaller, G., Schaerer, D., Meynet, G., \& Maeder, A. 1992, A\&AS, 96, 269

Schröder, C., \& Schmitt, J. H. M. M. 2007, A\&A, 475, 677

Seaton, M. J., Zeippen, C. J., Tully, J. A., et al. 1992, Rev. Mex. Astron. Astrofis., 23, 19

Shulyak, D., Tsymbal, V., Ryabchikova, T., Stütz Ch., \& Weiss, W. W. 2004, A\&A, 428, 993

Shulyak, D., Kochukhov, O., \& Khan, S. 2008, A\&A, 487, 689

Shulyak, D., Krtička, J., Mikulášek, Z., et al. 2010a, 524, A66

Shulyak, D., Ryabchikova, T., Kildiyarova, R., \& Kochukhov, O. 2010b, A\&A, 520, A 88

Škoda, P. 2008, Astronomical Spectroscopy and Virtual Observatory, ed. M. Guainazzi and P. Osuna (ESA), 97

Slettebak, A. 1949, ApJ, 110, 498

Smith, M. A., \& Groote, D. 2001, A\&A, 372, 208

Sokolov, N. A. 2000, A\&A, 353, 707

Sokolov, N. A. 2006, MNRAS, 373, 666

Sokolov, N. A. 2010, Ap\&SS, 330, 37

Stępień, K. 1978, A\&A, 70, 509

Stępień, K. 1998, A\&A, 337, 754

Townsend, R. H. D. Owocki, S. P., \& Groote D. 2005, ApJ, 630, L81

Trigilio, C., Leto, P., Leone, F., Umana, G., \& Buemi, C. S. 2000, A\&A, 362, 281
Trigilio, C., Leto, P., Umana, G., Buemi, C. S., \& Leone, F. 2008, MNRAS, 384, 1437

Trigilio, C., Leto, P., Umana, G., Buemi, C. S., \& Leone, F. 2011, ApJ, 739, L10

Tully, J. A., Seaton, M. J., \& Berrington, K. A. 1990, J. Phys. B, 23, 3811

van Leeuwen, F. 2007, A\&A, 474, 653

Vauclair, S. 2003, Ap\&SS, 284, 205

Vauclair, S., Dolez, N., \& Gough, D. O. 1991, A\&A, 252, 618

von Zeipel, H. 1924, MNRAS, 84, 665

Wamsteker, W., Skillen, I., Ponz, J. D., et al. 2000, Ap\&SS, 273, 155

Weiss, W. W., Albrecht, R., \& Wieder, R. 1976, A\&A, 47, 423

\section{Appendix A: Long tables}

Table A.1. List of the IUE observations of CU Vir.

\begin{tabular}{lccc}
\hline \hline Camera & Image & $\begin{array}{c}\text { Julian date } \\
2,400,000+\end{array}$ & Phase \\
\hline LWR & 3444 & 43883.94545 & 0.207 \\
LWR & 3445 & 43883.98646 & 0.286 \\
LWR & 3446 & 43884.03179 & 0.373 \\
LWR & 3447 & 43884.07508 & 0.456 \\
LWR & 3448 & 43884.11588 & 0.535 \\
LWR & 4044 & 43949.80082 & 0.687 \\
LWR & 4045 & 43949.84187 & 0.766 \\
LWR & 4046 & 43949.88284 & 0.844 \\
LWR & 4047 & 43949.92253 & 0.921 \\
LWR & 4048 & 43949.96366 & 1.000 \\
SWP & 3864 & 43883.91128 & 0.142 \\
SWP & 3865 & 43883.95163 & 0.219 \\
SWP & 3866 & 43883.99343 & 0.300 \\
SWP & 3867 & 43884.03658 & 0.382 \\
SWP & 3868 & 43884.07952 & 0.465 \\
SWP & 3869 & 43884.12081 & 0.544 \\
SWP & 4670 & 43949.80501 & 0.695 \\
SWP & 4671 & 43949.84709 & 0.776 \\
SWP & 4672 & 43949.88714 & 0.853 \\
SWP & 4673 & 43949.92677 & 0.929 \\
SWP & 4674 & 43949.96918 & 0.010 \\
\hline & & &
\end{tabular}


Table A.2. Observed and simulated effective semiamplitudes of light curves and parameters describing their forms in individual photometric bands of ultraviolet and optical regions (see Eq. (7)).

\begin{tabular}{|c|c|c|c|c|c|c|}
\hline \multirow[t]{2}{*}{ 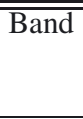 } & \multirow{2}{*}{$\begin{array}{c}\lambda \\
{[\AA]}\end{array}$} & \multicolumn{3}{|c|}{ "Observation } & \multicolumn{2}{|c|}{ Simulation } \\
\hline & & $N$ & $\begin{array}{c}A(\lambda) \\
{[\mathrm{mmag}]}\end{array}$ & $\psi(\lambda)$ & $\begin{array}{c}A(\lambda) \\
{[\mathrm{mmag}]}\end{array}$ & $\psi(\lambda)$ \\
\hline & 1230 & & & & 333 & -1.04 \\
\hline & 1260 & 11 & 479(15) & $-0.97(2)$ & 456 & -1.00 \\
\hline & 1290 & 11 & $455(14)$ & $-0.99(2)$ & 343 & -1.01 \\
\hline & 1320 & 11 & 285(9) & $-0.97(2)$ & 235 & -1.01 \\
\hline & 1350 & 11 & $233(5)$ & $-1.01(2)$ & 168 & -1.04 \\
\hline & 1380 & 11 & $312(7)$ & $-0.99(2)$ & 180 & -1.03 \\
\hline & 1410 & 11 & $323(7)$ & $-1.00(2)$ & 204 & -1.03 \\
\hline & 1440 & 11 & 251(4) & $-1.02(1)$ & 108 & -1.06 \\
\hline & 1470 & 11 & 193(5) & $-1.03(2)$ & 82 & -1.03 \\
\hline & 1500 & 11 & 159(5) & $-1.01(2)$ & 56 & -1.04 \\
\hline & 1530 & 11 & 194(4) & $-1.01(2)$ & 98 & -0.97 \\
\hline & 1560 & 11 & $185(5)$ & $-1.00(2)$ & 79 & -0.84 \\
\hline & 1590 & 11 & $125(5)$ & $-0.89(3)$ & 40 & -0.58 \\
\hline & 1620 & 11 & $75(5)$ & $-0.77(4)$ & 24 & -0.24 \\
\hline & 1650 & 11 & $63(5)$ & $-0.73(5)$ & 26 & -0.05 \\
\hline & 1680 & 11 & $52(5)$ & $-0.65(5)$ & 21 & 0.30 \\
\hline & 1710 & 11 & $62(4)$ & $-0.72(4)$ & 27 & -0.31 \\
\hline & 1740 & 11 & 71(5) & $-0.72(4)$ & 24 & 0.10 \\
\hline & 1770 & 11 & 97(4) & $-0.75(3)$ & 22 & 0.46 \\
\hline & 1800 & 11 & $57(5)$ & $-0.74(5)$ & 30 & 0.71 \\
\hline & 1830 & 11 & $40(5)$ & $-0.70(8)$ & 25 & 0.58 \\
\hline & 1860 & 11 & $26(4)$ & $-0.48(9)$ & 23 & 0.66 \\
\hline & 1890 & 11 & $34(5)$ & $-0.71(8)$ & 22 & 0.38 \\
\hline & 1920 & & & & 25 & 0.63 \\
\hline & 1950 & & & & 28 & 0.80 \\
\hline & 1980 & & & & 41 & 0.96 \\
\hline & 2010 & 10 & 77(4) & $0.35(4)$ & 36 & 0.93 \\
\hline & 2050 & 10 & 66(4) & $0.47(4)$ & 41 & 0.96 \\
\hline & 2100 & 10 & $73(4)$ & $0.59(3)$ & 43 & 1.00 \\
\hline & 2150 & 10 & $77(5)$ & $0.50(4)$ & 42 & 1.00 \\
\hline & 2200 & 10 & $87(5)$ & $0.62(4)$ & 42 & 1.01 \\
\hline & 2250 & 28 & $42(3)$ & $0.69(3)$ & 27 & 0.81 \\
\hline & 2300 & 10 & $57(5)$ & $0.46(5)$ & 25 & 0.78 \\
\hline & 2350 & 10 & $63(3)$ & $0.10(3)$ & 24 & -0.18 \\
\hline & 2400 & 10 & $56(5)$ & $0.07(5)$ & 24 & -0.14 \\
\hline & 2450 & 10 & 49(4) & $0.39(5)$ & 16 & 0.44 \\
\hline & 2500 & 28 & 25(3) & $0.64(5)$ & 16 & 0.70 \\
\hline & 2550 & 10 & $43(2)$ & $0.56(3)$ & 22 & 0.86 \\
\hline & 2600 & 10 & $41(3)$ & $0.58(4)$ & 21 & 0.84 \\
\hline & 2650 & 10 & $50(4)$ & $0.78(4)$ & 29 & 1.01 \\
\hline & 2700 & 10 & $48(2)$ & $0.85(3)$ & 29 & 1.03 \\
\hline & 2750 & 10 & $47(3)$ & $0.75(3)$ & 20 & 0.89 \\
\hline & 2800 & 10 & $65(3)$ & $0.90(3)$ & 39 & 1.10 \\
\hline & 2850 & 10 & $60(3)$ & $0.91(3)$ & 29 & 1.05 \\
\hline & 2900 & 10 & $58(2)$ & $1.04(2)$ & 33 & 1.12 \\
\hline & 2950 & 10 & $61(2)$ & $1.05(2)$ & 41 & 1.13 \\
\hline & 2990 & 10 & $69(2)$ & 1.10(1) & 44 & 1.14 \\
\hline & 3300 & 38 & $75(3)$ & 1.21(2) & & \\
\hline$u$ & 3500 & 986 & $76.9(3)$ & $1.22(1)$ & 45 & 1.13 \\
\hline$U$ & 3600 & 738 & $70.1(5)$ & $1.19(1)$ & 45 & 1.13 \\
\hline$v$ & 4100 & 1130 & $42.4(2)$ & $0.95(1)$ & 34 & 1.11 \\
\hline$B$ & 4400 & 992 & 43.1(3) & 0.99(1) & 33 & 1.11 \\
\hline$b$ & 4650 & 1059 & $41.5(2)$ & $1.00(1)$ & 31 & 1.11 \\
\hline$\beta$ & 4860 & 95 & $41(2)$ & $1.06(3)$ & & \\
\hline$H p$ & 5100 & 112 & $37.8(9)$ & 1.01(2) & 31 & 1.11 \\
\hline$y+V$ & 5500 & 2251 & $33.2(2)$ & $1.07(1)$ & 25 & 1.11 \\
\hline$R$ & 7530 & 32 & $30(4)$ & $1.12(8)$ & & \\
\hline
\end{tabular}

Notes. $N$ denotes number of observations. 